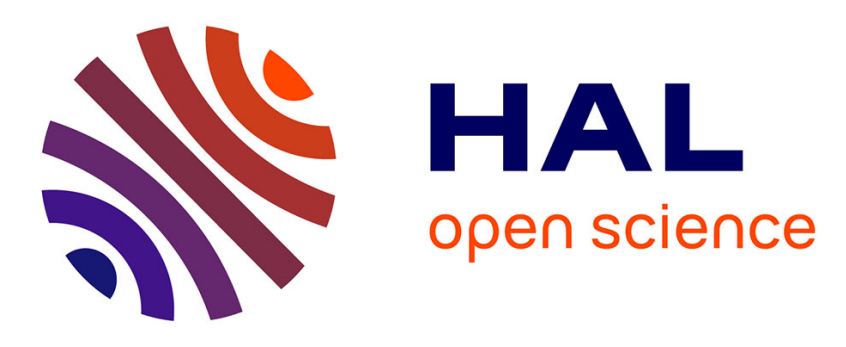

\title{
Kinetic Target-Guided Synthesis: Reaching the Age of Maturity
}

Damien Bosc, Virgyl Camberlein, Ronan Gealageas, Omar Castillo-Aguilera, Benoit Deprez, Rébecca F. Déprez-Poulain

\section{- To cite this version:}

Damien Bosc, Virgyl Camberlein, Ronan Gealageas, Omar Castillo-Aguilera, Benoit Deprez, et al.. Kinetic Target-Guided Synthesis: Reaching the Age of Maturity. Journal of Medicinal Chemistry, 2019, 63 (8), pp.3817-3833. 10.1021/acs.jmedchem.9b01183 . hal-03051818

\section{HAL Id: hal-03051818 https://hal.science/hal-03051818}

Submitted on 10 Dec 2020

HAL is a multi-disciplinary open access archive for the deposit and dissemination of scientific research documents, whether they are published or not. The documents may come from teaching and research institutions in France or abroad, or from public or private research centers.
L'archive ouverte pluridisciplinaire HAL, est destinée au dépôt et à la diffusion de documents scientifiques de niveau recherche, publiés ou non, émanant des établissements d'enseignement et de recherche français ou étrangers, des laboratoires publics ou privés. 


\section{Kinetic Target-Guided Synthesis: reaching the age of maturity}

Damien Bosc, ${ }^{\dagger}$ Virgyl Camberlein, ${ }^{\dagger}$ Ronan Gealageas, ${ }^{\dagger}$ Omar Castillo-Aguilera, ${ }^{\dagger}$ Benoit Deprez, ${ }^{\dagger}$ Rebecca Deprez-Poulain ${ }^{*},+, \ddagger$

† Univ. Lille, Inserm, Institut Pasteur de Lille, U1177 - Drugs and Molecules for Living Systems, F-59000 Lille, France;

‡ Institut Universitaire de France, F- 75005, Paris, France.

\section{Keywords:}

KTGS, triazole, fragment, in situ click chemistry, hit-to-lead, lead-optimization

\section{Abstract}

Kinetic target-guided synthesis (KTGS) is an original discovery strategy allowing a target to catalyze the irreversible synthesis of its own ligands from a pool of reagents. Though pioneered almost two decades ago, it only recently proved its usefulness in medicinal chemistry, as exemplified by the increasing number of protein targets used, the wider range of target and pocket types, and the diversity of therapeutic areas explored. In recent years, two new leads for in vivo studies were released. Amidations and multicomponent reactions expanded the armamentarium of reactions beyond triazole formation and two new examples of in cellulo KTGS were also disclosed. Herein, we analyze the origins and the chemical space of both KTGS ligands and warhead-bearing reagents. We review the KTGS timeline focusing on recent cases in order to give medicinal chemists the full scope of this strategy which has great potential for hit discovery and hit or lead optimization.

\footnotetext{
*Corresponding author : rebecca.deprez@univ-lille.fr; $\underline{w w w . d e p r e z l a b . f r}$
} 


\section{INTRODUCTION}

\section{Definitions and terminology}

Protein-templated synthesis (PTS) or Target-guided synthesis (TGS) of ligands use the protein of interest as a template to form its own ligands (Figure 1) $)^{1-2}$. Although proteintemplated synthesis of a ligand has been unexpectedly observed by some biochemists in the past, it is a rather novel concept that has gained a real interest in drug discovery labs in the past decade. As a consequence, it has been highlighted as part of bioorthogonal chemistryinspired advances in medicinal chemistry. ${ }^{3}$ Prior to analyzing the recent achievements in the field of Kinetic-Target Guided Synthesis (KTGS), one of the protein-templated strategies for ligand discovery, the terminology for these strategies will briefly be reviewed.

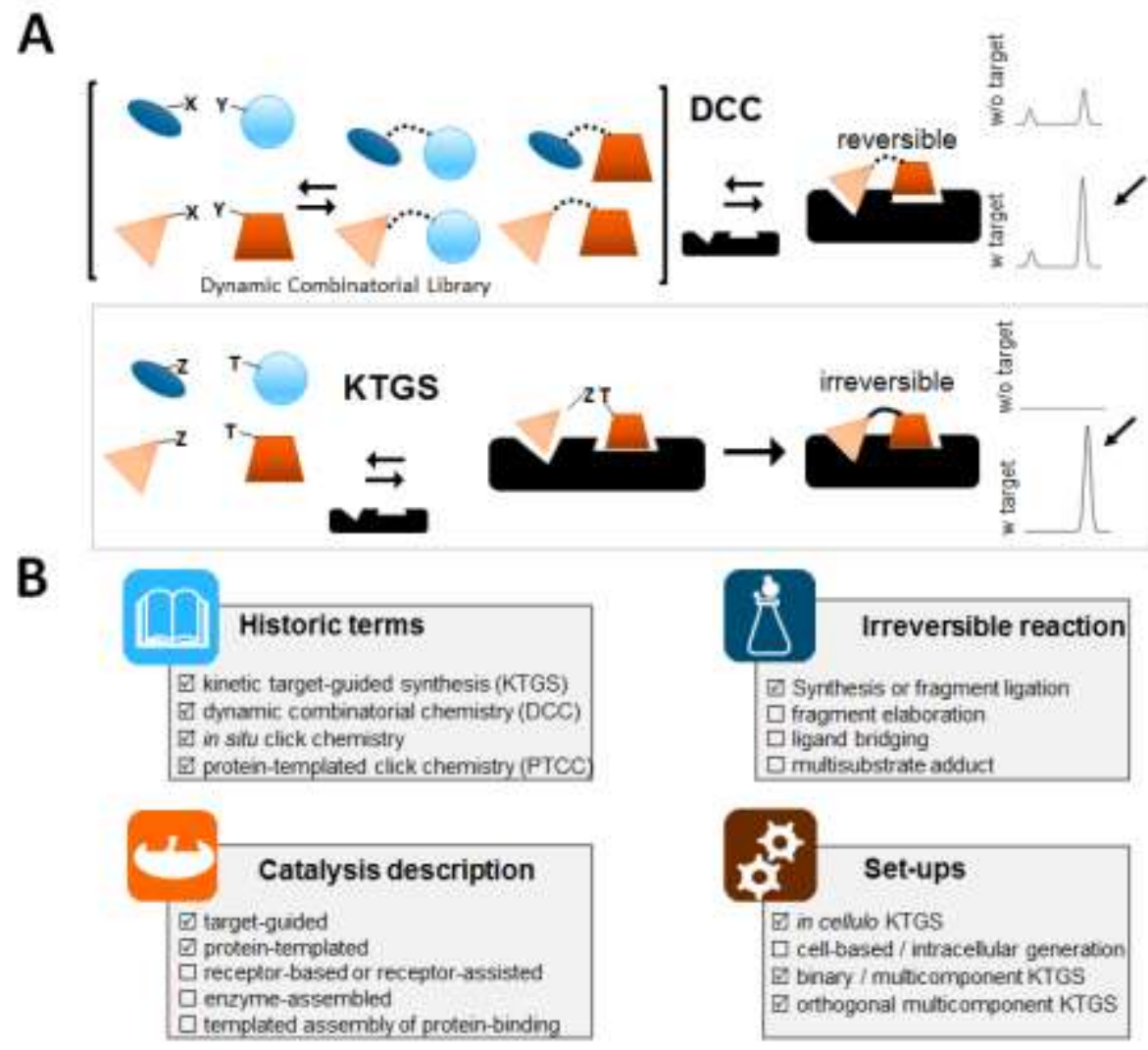

Figure 1: Principles and terminology for target-guided synthesis. ${ }^{a}$

a preferred terms are checked. (A) i. Principle of Dynamic Combinatorial Chemistry (DCC) where the target shifts the equilibrium towards the most affine ligand. ii. Principle of Kinetic Target Guided synthesis (KTGS) where the target brings in close proximity the affine reagents so that they can irreversibly react to form the final ligand. (B) Wording for KTGS experiments in the literature and proposed terminology (checkbox).

The different approaches for PTS are classified as thermodynamically or kinetically controlled synthesis. ${ }^{4}$ Kinetic target guided synthesis (KTGS) and dynamic combinatorial 
chemistry (DCC) (Figure 1A) stand respectively for the protein-templated synthesis of ligands by irreversible or reversible reactions. In DCC, there is a continuous interconversion between the reagents and the final products (dynamic combinatorial library, Figure $1 \mathrm{~A}$ ). The target shifts the thermodynamic equilibrium between products and reagents towards ligands that have the highest affinity for the target. ${ }^{5,6}$ These ligands are produced in significantly high amounts in the presence of the protein. In KTGS, the targets bring in close proximity with theright orientation, affine reagents, so that they react irreversibly. ${ }^{2,7-8}$ In situ click chemistry, first disclosed for the discovery of acetylcholinesterase (AChE) inhibitors, is the most popular kinetic target-guided synthesis. It allows protein-templated triazole synthesis from biocompatible alkynes and azides. $^{9}$

A review of the literature shows that the term "target-guided synthesis" (TGS) has sometimes been replaced with synonyms like "receptor-based"-, "receptor-assisted"- or "protein-templated"-synthesis (Figure 1B). Several groups used "combinatorial chemistry", in the original sense of mixing arrays of reagents with the same reactivity, without mentioning the type of protein-templated reaction. We recommend using "combinatorial chemistry" along with "kinetic" or "dynamic" terms where appropriate. Three different set ups for KTGS reactions are reported and have been defined earlier²: a binary format, a multicomponent format where clusters of reagents are used as mixtures or an orthogonal multicomponent format.

Recent discussions question whether target-guided strategies belong to fragmentbased drug discovery (FBDD) techniques. Indeed, the term "fragment" is sometimes used to describe biocompatible reagents for protein-templated reactions (Figure 1B). Fragments were initially defined as small compounds that comply to the rule-of-three (Ro3). ${ }^{10}$ This Ro3 was later adjusted. Some reports showed that it should be applied in a context-dependent manner, more specifically according to to hydrogen bond donors and acceptors thresholds. ${ }^{11}$ FBDD is based on the identification of weakly bound fragments which are further optimized into final ligands upon linking or growing. Conversely, TGS is based on the identification of high-affinityligands (ie final compounds) from in situ ligations. We thus recommend to use "fragment" when reagents for KTGS are discovered by fragment screening. The term "reagents" can be used to 
describe the chemical precursors for KTGS and "warhead-bearing reagents" can be used for precursors that help to drive the final ligand within a protein pocket (either previously known or new).

Several teams have paved the way to target-guided synthesis in cells. To include these, we propose to add the "in cellulo" prefix to target-guided or to protein templated synthesis, and to avoid the phrase "in cellulo click chemistry" that cannot sufficiently distinguish between the protein labelling using click chemistry for chemical biology purposes, and the genuine KTGS in cells.

\section{History and recent achievements}

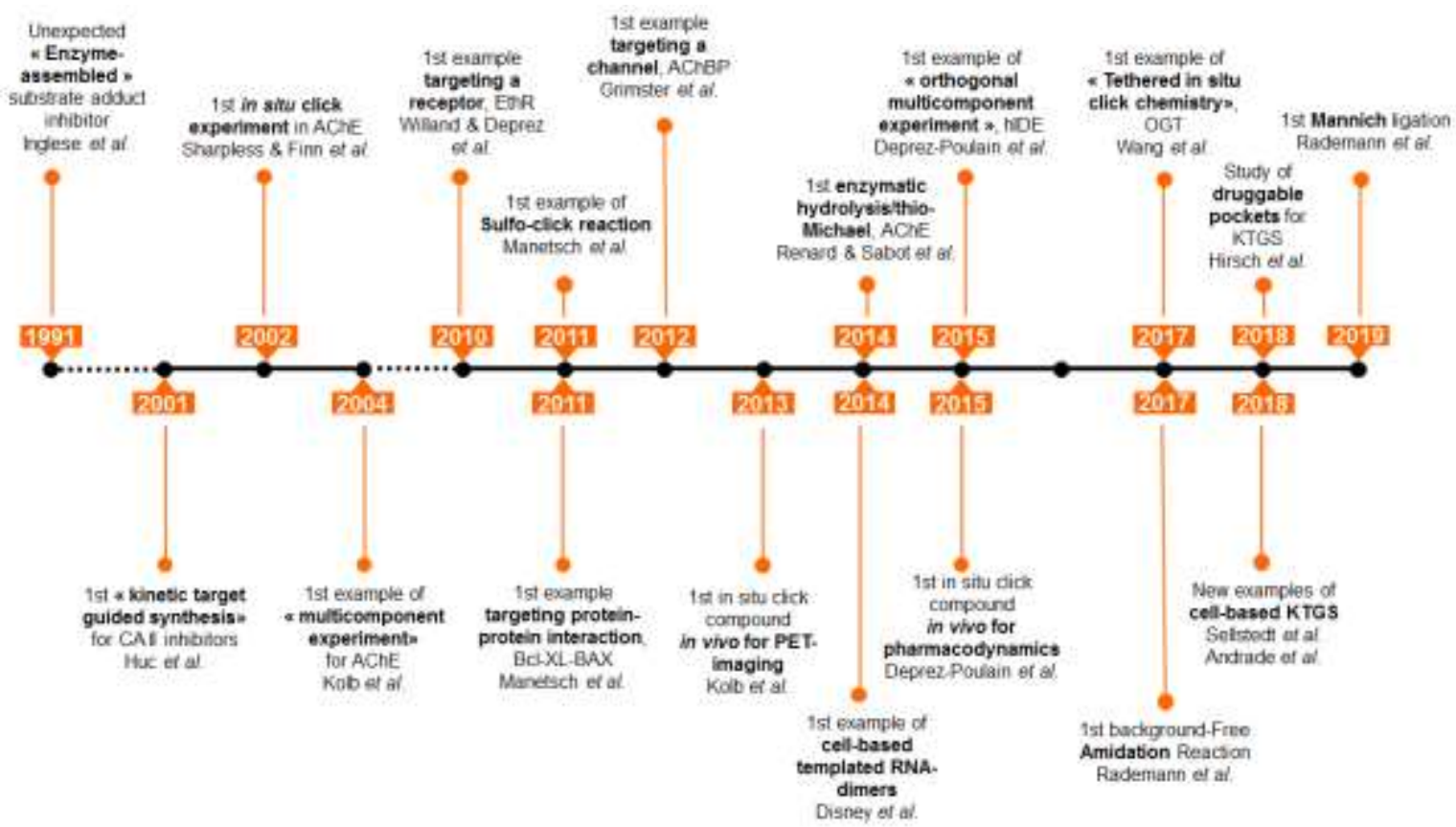

Figure 2 : Timeline for KTGS and in situ click chemistry development \& use.

The main events in the timeline of Kinetic Target-Guided Synthesis strategies are presented in Figure 2. The first, although unexpected, example of a KTGS reaction was disclosed in 1991 by Inglese et al: an inhibitor of glycinamide ribonucleotide transformylase (GARTfase) was templated from $\beta$-glycinamide ribonucleotide substrate and $\mathrm{N} 10$ (bromoacetyl)-DDF an affinity label inspired by the enzyme cofactor. ${ }^{12}$ Similarly, several drugs can be considered as KTGS precursors as they react with co-factors within their target enzyme to provide an adduct that is the active species. Examples include: carbidopa, that reacts with 
pyridoxal phosphate to inhibit DOPA decarboxylase and selegilin that reacts with FAD to block MAO-B and isoniazid reacts with NAD to inhibit MtB catalase. ${ }^{13}$ Ataluren (PTC-124), a recent drug used for its nonsense codon suppression activity in the treatment of Duchenne's disease, was initially a false positive in a reporter gene assay that used firefly luciferase (FLuc). It was shown later that FLuc acts as a template for the formation of its own strong inhibitor PTC124$\operatorname{AMP}\left(\mathrm{IC}_{50}=10 \mathrm{nM}\right)$ from ataluren and ATP. ${ }^{14}$

The first use of KTGS for medicinal chemistry was disclosed by Huc et al in 2001. They describe the thiol alkylation using various halides in the presence of human carbonic anhydrase II (hCAII). ${ }^{15}$ In 2002, following the disclosure of copper catalyzed click reactions by both Sharpless' and Meldal's teams ${ }^{16-17}$, "in situ click-chemistry" was coined and was applied for the first time to the model protein acetylcholinesterase. ${ }^{9}$ Since then, enzymes but also other target families like transcriptional factors ${ }^{18-19}$, channels ${ }^{20}$ or protein-protein interactions ${ }^{21}$, have been explored using KTGS (Suppl. Table 1). The drugability of the KTGS protein pockets has also been recently studied. ${ }^{22}$

The format of KTGS experiments itself evolved from binary to multicomponent approaches that were further completed within the orthogonal multicomponent setting ${ }^{2}$. As well, the set of KTGS compatible chemical reactions has been greatly expanded with the identification of reactions like the sulfoclick reaction by Manetsch's team ${ }^{21}$, the hydrolysis/thio-Michael coupling by Renard's team ${ }^{23}$, the amidation reaction ${ }^{24}$ and the Mannich reaction ${ }^{19}$ both by Rademann's team (Suppl. Table 2). In 2017, Wang et al. combined the covalent binding of a KTGS bifunctional precursor to the target with subsequent in situ click chemistry to discover new ligands for transferase ${ }^{25}$. Besides the increasing diversity of reactions, targeted protein types and KTGS formats, the biological characterization of templated ligands has also evolved. It now includes not only the in vitro activity on isolated targets but also cellular activities and more recently in vivo pharmacodynamics for imaging or therapy.

Efficient medicinal chemistry requires an optimal interplay between diversity generation and selection of structures with the best properties. KTGS has demonstrated in the past few years its potential to combine both. We review here the recent development and successful 
case studies, and provide an analysis of KTGS warhead-bearing reagents and ligands, to bring the medicinal chemists the full view of this promising drug discovery strategy and its added value over other diversity/selection methods.

\section{KTGS DESIGN AND PRACTICAL ASPECTS}

Our analysis of the literature points out that the main practical issues for KTGS, are the type of reactions, the detection methods and the choice of a good warhead-bearing reagent (Suppl. Table 2). ${ }^{2}$

\section{Chemical reactions}
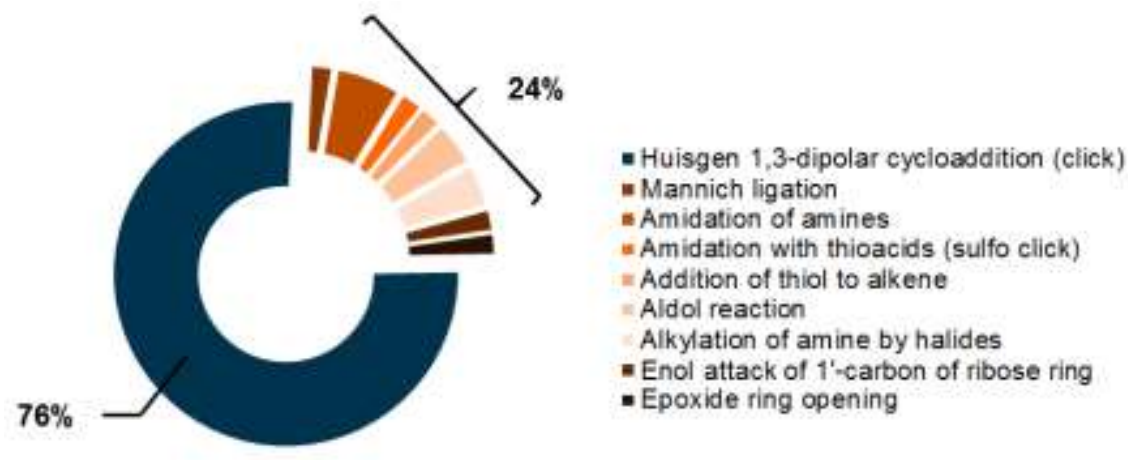

Figure 3 : KTGS reactions. ${ }^{\text {a }}$

a From published examples

Most KTGS reactions are Huisgen 1,3-dipolar cycloaddition between azides and alkynes, i.e. in situ click-chemistry. This reaction accounts for $76 \%$ of the target-guided synthesis experiments (Figure 3) and the proportion of KTGS ligands obtained by in situ clickchemistry is $81 \%$ of all templated ligands. This high proportion is directly linked to the biocompatibility of alkynes and azides and their commercial and synthetic availability. 


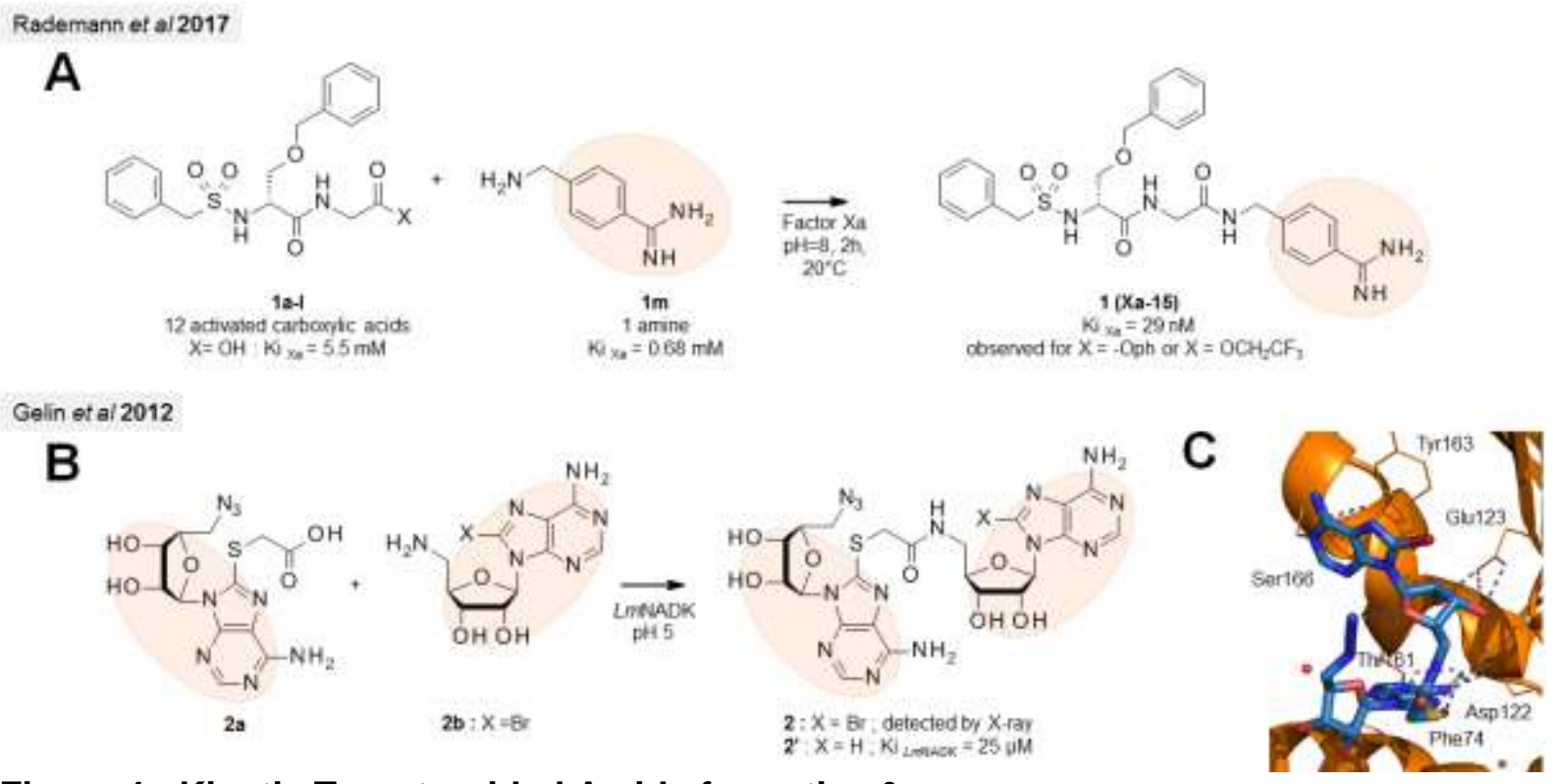

Figure 4 : Kinetic Target-guided Amide formation. ${ }^{\text {a }}$

a (A) KTGS amidation using activated carboxylic acid reagents produced factor Xa inhibitor $1 .{ }^{26}$; (B) KTGS amidation in LmNADK and subsequent synthetic optimization of ligand; (C) Binding of 2 (blue) in NAD pocket (orange) of $L m N A D K$ (PDB: $3 v 8 p$ ). Key warheads are highlighted in salmon.

Some other templated reactions involve nucleophilic attack of epoxides or Michael acceptors. Rademann et al recently published the first amidation KTGS reaction to obtain a Factor Xa inhibitor. ${ }^{24}$ An activated carboxylic acid promotes the templated reaction (Figure 4A). In particular, a - $\mathrm{OCH}_{2} \mathrm{CF}_{3}$ ester seems a better activated species as it does not produce any adduct with the amine in the absence of Factor $\mathrm{Xa}$, even with increased temperature or reaction time. ${ }^{24}$ This reaction could be widely used, as amide formation is still the most popular reaction in drug discovery ${ }^{27}$. Earlier, Gelin et al. had also described the KTGS templated formation of a dinucleoside inhibitor of the NADkinase from L.monocytogenes (LmNADK1), via an amide bond formation observed directly in X-ray co-crystals (Figure 4B and 4C). ${ }^{28}$

The first example of multicomponent reaction (MCR), previously published by Weber et al., used the Ugi reaction to identify inhibitors of thrombin (Figure 5A) ${ }^{29}$ Another published example of KTGS using MCR is the Mannich ligation between formaldehyde, an amine and some aza-heterocycles, which led to the discovery of STAT5 inhibitors (Figure 5B). ${ }^{19}$ 


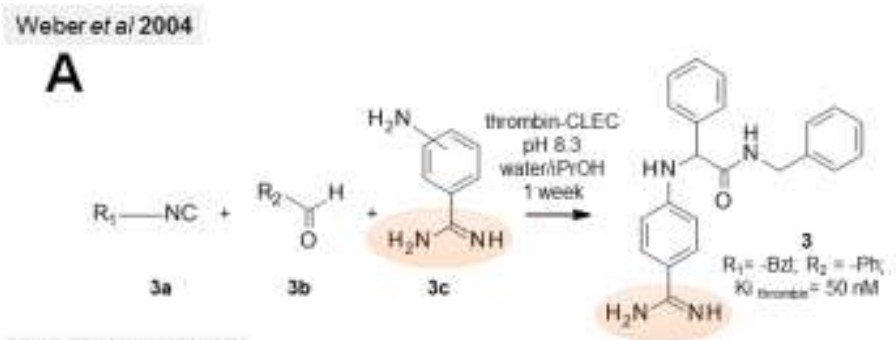

Rademann et al 2019

B

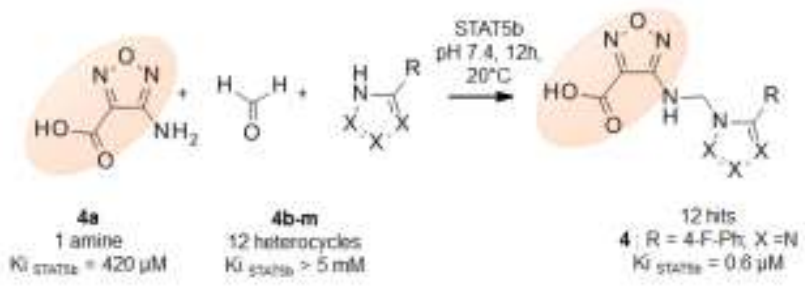

Figure 5 : Multicomponent reactions in KTGS. ${ }^{a}$

a (A) First example of an Ugi templated reaction by thrombin; (B) Mannich templated reaction by STAT5b from an amine fragment containing an amidine warhead. Warheads are highlighted in salmon. CLEC: crosslinked enzyme crystals.

Noteworthy, Renard et al reported in 2014 an elegant strategy that uses the enzymatic activity of the target itself to generate at the catalytic site, thiol reagents from S-acylated precursors that further react in a KTGS "in situ thio-Michael reaction" with acrylamides. ${ }^{23}$

\section{Warhead-bearing reagents}

A

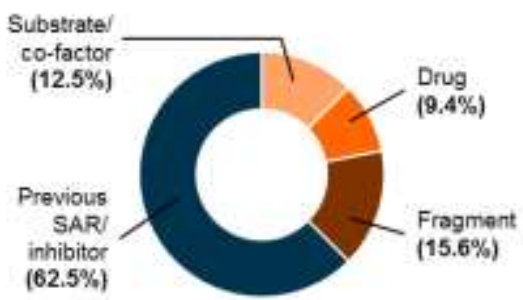

B

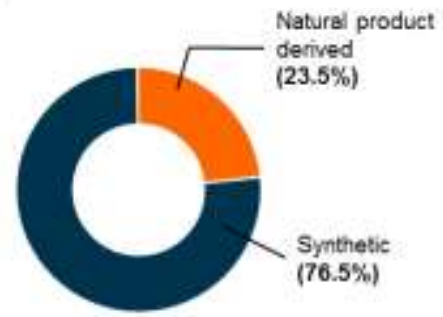

C

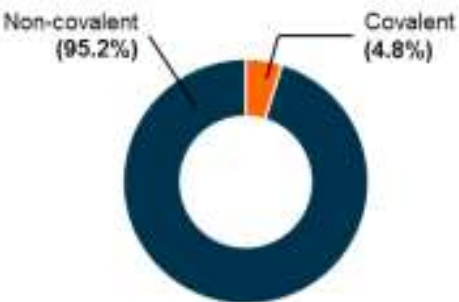

Figure 6 : Origin and nature of warhead-bearing reagents. ${ }^{a}$

a (A) Previous SAR account for $62.5 \%$ of warhead-bearing reagents origins; (B) More than $75 \%$ of warhead-bearing reagents are synthetic compounds. (C) Almost $5 \%$ of warhead-bearing reagents are bifunctional and thus lead to covalent KTGS ligands.

The selection and/or design of warhead-bearing reagents is crucial for the success of KTGS. An analysis of the published warhead-bearing reagents that led to KTGS ligands (Supp Table 1, Figure 6) shows that the main source of inspiration is known SAR or previously described inhibitors or modulators (62.5\%). For example, KTGS use a benzylsulfonamide warhead to target COX $-2,{ }^{30}$ a hydroxamate warhead to target metalloproteases, ${ }^{31}$ a guanidine 
warhead to target Factor $\mathrm{Xa}^{24}$. Two different cognate ligands can also be used to produce bivalent ligands. For example, AChE inhibitors were discovered from derivatives of tacrine or huprine, that are catalytic site ligands, coupled to derivatives of phenanthridinium and 1,2,3,4tetrahydroisoquinolines, that are peripheral site ligands..$^{32-33}$

More than $12 \%$ of warhead-bearing reagents for enzyme-templated KTGS are inspired by the substrate or co-factor, like biotin ${ }^{34,35}$ or $\mathrm{NAD}^{28}$. A few warhead-bearing reagents were directly derived from drugs (9.4\%) that are exclusively natural compounds: kanamycin ${ }^{36}$ and solithromycin ${ }^{37}$. Among the reagents inspired by previous SAR $(62.5 \%)$ some were highly inspired by drugs or clinical candidates like celecoxib ${ }^{30}$ for COX2 inhibitors, rupintrivir $(A G 7088)^{38}$ for enteroviral protease inhibitors or navitoclax (ABT-263) ${ }^{21}$ for Bcl inhibitors. More than $15 \%$ of KTGS ligands come from reagents that were identified by fragment screening, such as endothiapepsin inhibitors, ${ }^{39}$ O-GlcNAc transferase inhibitors, ${ }^{25}$ myelin-associated glycoprotein ligands ${ }^{40}$ or STAT5 inhibitors ${ }^{19}$. Out of all templated ligands, $23.5 \%$ derive from nature-inspired warhead-bearing reagents (Figure 6B). A few teams designed genuine covalent inhibitors (4.8\%, Figure $6 \mathrm{C}$ ) by targeting cysteines during KTGS, discovering thus potent inhibitors of enteroviral protease ${ }^{38}$ or O-GIcNAc transferase ${ }^{25}$. In this context, the warhead-bearing reagents must be bifunctional.

KTGS and other protein-templated reactions have sometimes been classified as an extension of fragment linking strategies. We thus analyzed both the distribution of the calculated parameters and structural features of reagents used for KTGS and compared these distribution with those of fragments (Figure 7 and 8). First, most of the reagents used for KTGS, and warhead-bearing reagents in particular, do not comply to the Rule of 3 (Ro3) for fragments (Figure 7). For example, more than $75 \%$ display a number of $\mathrm{HBD}<=3$, and $70 \%$ a cLogP $<3$, and $62 \%$ have a molecular weight higher than $300 \mathrm{~g} / \mathrm{mol}$ (Figure $7 \mathrm{~A}, \mathrm{~B}$ and $\mathrm{C}$ ). This is especially true for warhead-bearing reagents inspired from drugs and substrates. As it has been pointed out before, HBA is the most affected parameter. ${ }^{11}$ Indeed, only $29 \%$ of these reagents display a number of $\mathrm{HBA}<=3$, and they are exclusively non-azide (Figure $7 \mathrm{D}$ and $\mathrm{E}$ respectively). In all, $82 \%$ of warhead-bearing reagents used in KTGS violate the Ro3. 

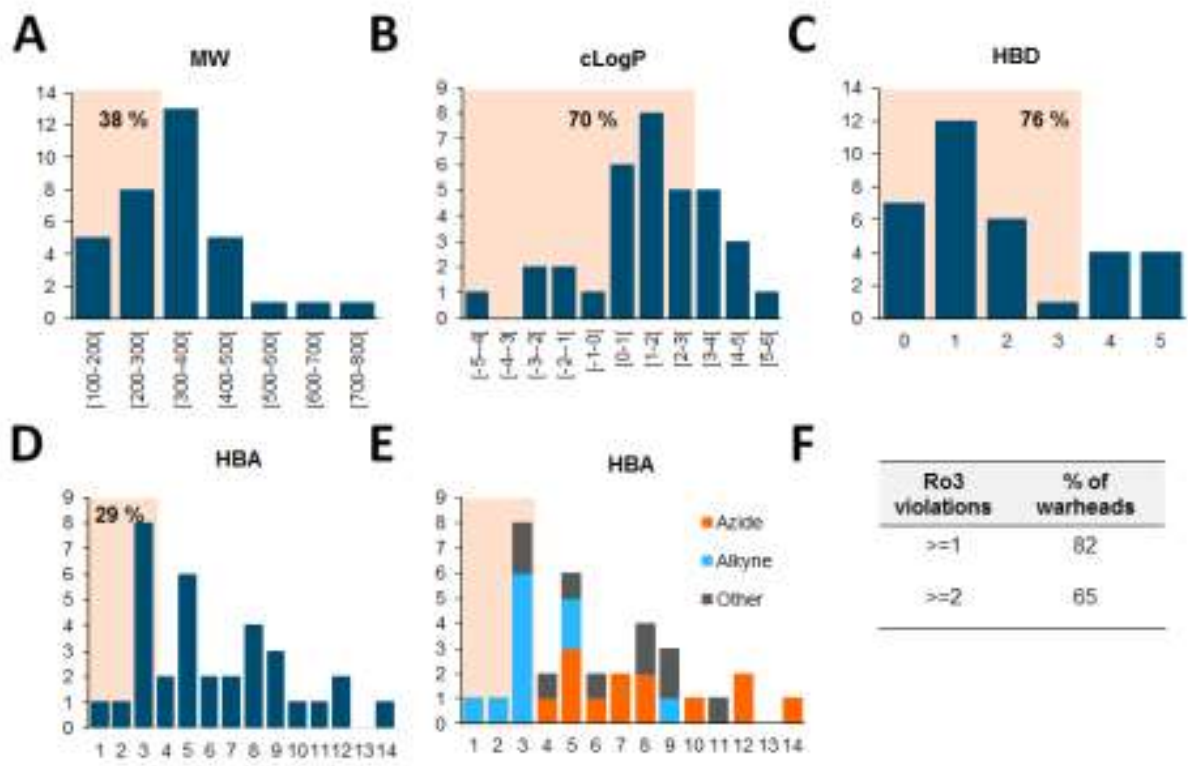

Figure 7 : Counts of MW, cLogP, HBA, HBD for warhead-bearing reagents. ${ }^{a}$

${ }^{a}$ In salmon, the proportion of warhead-bearing reagents that fulfil the Ro3 limit for a given count. (A) MW in $\mathrm{g} / \mathrm{mol}$, (B) cLogP, (C) HBD, (D) HBA, (E) HBA sorted by reactive chemical moiety, (F) Ro3 violations. In total only $18 \%$ of warhead-bearing reagents fulfill all the Ro3 critera. The most stringent parameter being HBA.

\section{A}

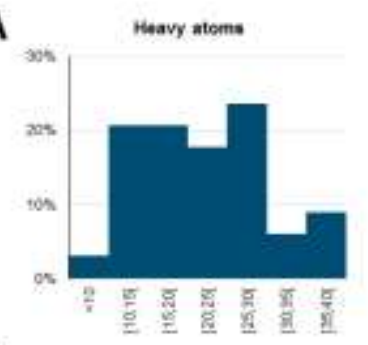

D

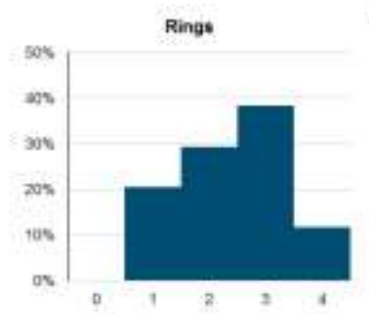

B

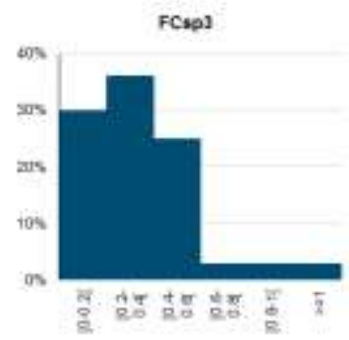

$\mathbf{E}$

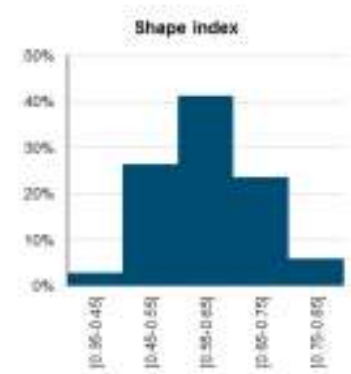

C

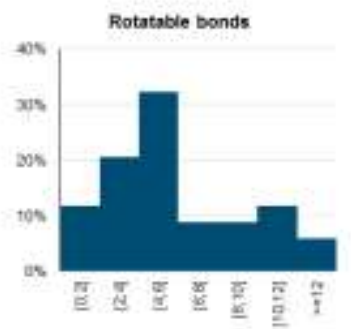

$\mathbf{F}$

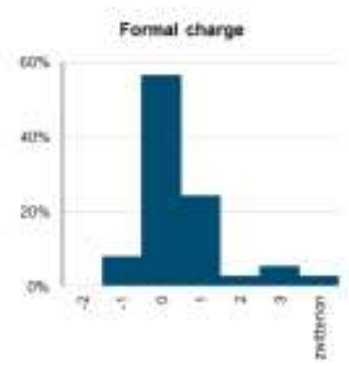

Figure 8 : Structural features of warhead-bearing reagents. ${ }^{a}$

a Distribution ( $N=34$ ) of the (A) number of heavy atoms, (B) fraction of sp3 hybridized carbon atoms (FCsp3), (C) number of rotatable bonds, (D) number of rings, $(E)$ shape index, $(F)$ formal charges.

Examination of structural features shows that about $82 \%$ of warhead-bearing reagents contain between 10 and 30 heavy atoms (Figure 8A). Extreme values are displayed by natural compounds like aminoglycosides ${ }^{36}$, ketolides $^{37}$, cyclopeptides ${ }^{41}$ or substrates adducts. In comparison, $81 \%$ of fragments, for which a fragment-protein complex is available in the PDB, display a number of heavy atoms between 10 and $16 .{ }^{42}$ About $90 \%$ of warhead-bearing reagents have a limited fraction of sp3-hybridized carbon atoms (Fsp3 $<0.6$, Figure 8B) like 
fragments $\left(90 \%\right.$ with Fsp3 < 0.5).$^{42}$ Half of warhead-bearing reagents display of a higher rotatable bonds number ( $53 \%$ between 2 and 6 , Figure $8 \mathrm{C})$ and more rings $(50 \%$ have more than 2 rings, Figure $8 D$ ), than fragments. ${ }^{42}$ Their distribution of molecular shape index (spherical $<0.5<$ linear) is centered at $0.55 \pm 0.05(41.1 \%$, Figure $8 \mathrm{E})$. Half of the reagents have a formal charge of $0,32 \%$ are positively charged, only $8 \%$ display negative charges and $3 \%$ are zwitterionic (Figure $8 F$ ). In contrast, Shaw et al. showed a higher proportion of neutral compounds (68\%), and twice more abundant negatively charged $(22 \%)$ than positively charged compounds (11\%) within a set of 462 fragments. ${ }^{42}$ Finally, reagents for KTGS usually display a potency or binding equal or below $500 \mu \mathrm{M}$ while fragments usually bind the target at a millimolar concentration. KTGS is not thus exclusively a fragment linking strategy as it requires at least one reagent binding in an affinity range beyond the one of classical fragments.

\section{Formats, sizes, detection methods and hit rates}
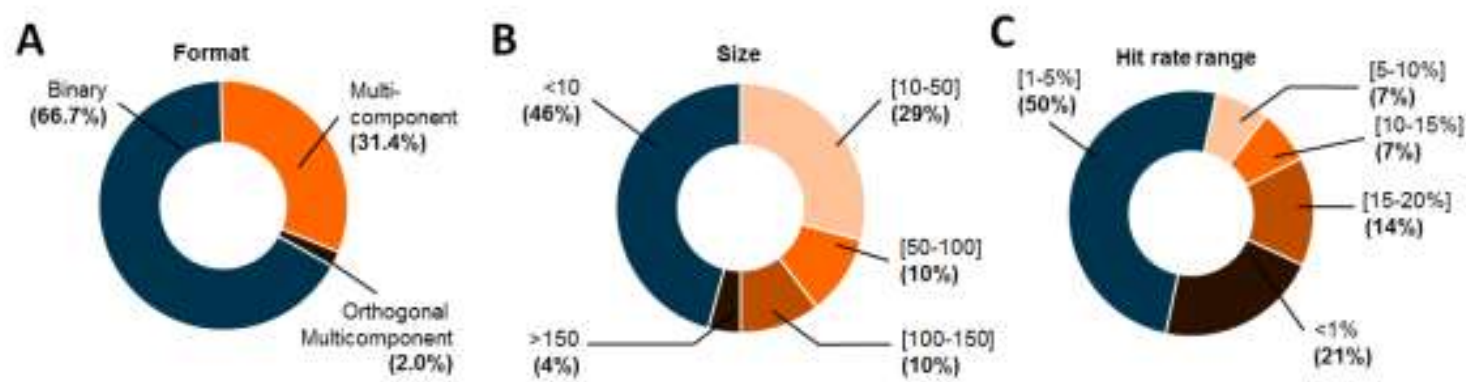

Figure 9 : Format, size and hit rate of KTGS. ${ }^{a}$

a (A) Format types of reported KTGS, (B) Size of the KTGS experiment expressed as the number of putative ligands. $25 \%$ of KTGS experiments size $>50$ putative ligands. (C) Proportion of KTGS with a given hit rate, restricted to KTGS with a size $>30$ putative ligands.

Three different types of formats for KTGS exist: either binary, multicomponent or orthogonal multicomponent. ${ }^{2} \mathrm{~A}$ high majority of the KTGS publications report binary formats (67\%, Figure $9 \mathrm{~A})$ and a small library size of potential ligands. The multicomponent format (not to be mistaken for multi-component reactions) uses clusters of reagents instead of pairs of reagents and thus allows to access a larger number of putative ligands, exploiting the full potential of a kinetically driven competition between a large number of reagents. $14 \%$ of KTGS examples describe the exploration of more than 100 putative ligands (Figure $9 \mathrm{~B}$ ). At last, in situ click-chemistry may produce 2 different regio-isomers (1,4- or 1,5-triazoles) with very 
different binding capabilities, from the same set of reagents. This thus doubles the number of possible ligands.

There are differences in the size of KTGS experiments, expressed as the number of possible ligands. As a result, hit-rates range from $0.2 \%$ to $100 \%$. Since many KTGS experiments have a limited size (less than 10 possible ligands for $46 \%$ of the examples Figure 9B) and are inspired from previously known SAR, some show a $100 \%$ hit-rate. Among the KTGS experiments with a minimum size of 30 possible ligands, $50 \%$ show a hit rate comparable to random screening (hit rate $=1-5 \%$ ) (Figure 9C). Usually, for larger KTGS, the diversity is carried by only one type of reagents (not the warhead-bearing reagent). For example, Kwarcinski et al. disclosed a KTGS with c-Src, a nonreceptor tyrosine kinase from one thiol and 110 commercial acrylamide fragments resulting in $4 \%$ hit rate (110 possible ligands). ${ }^{43}$ IDE inhibitors were discovered from 90 diverse alkynes and 2 azides (360 putative ligands, $18.3 \%$ hit rate). ${ }^{31} h \mathrm{DAO}$ inhibitors were obtained from 1 alkyne and 250 diverse azides (500 putative ligands and $0.2 \%$ hit rate). ${ }^{44}$

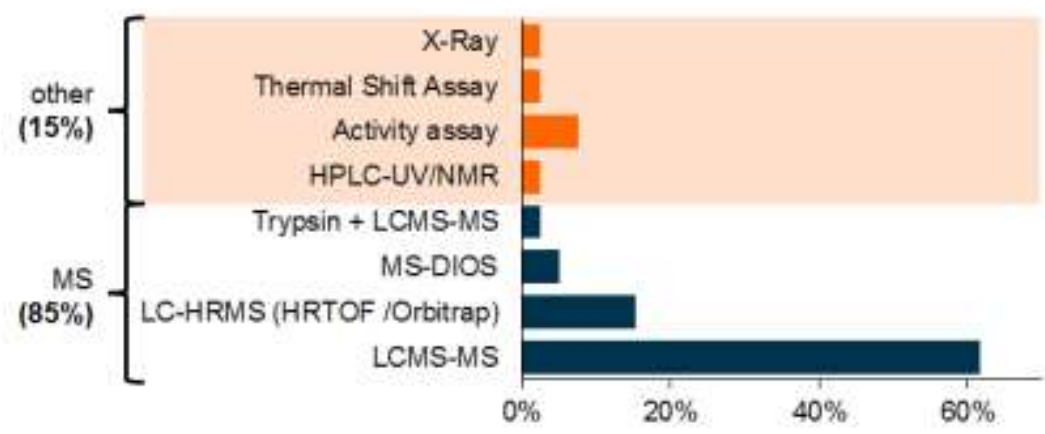

Figure 10 : Templated ligand detection methods. ${ }^{\text {a }}$

$85 \%$ of detection methods of ligand rely on mass spectrometry.

Mass-spectrometry-based protocols account for $85 \%$ of detection methods (Figure 10). In recent years, several teams have described the use of high-resolution MS to confirm the molecular formula of KTGS ligands and to increase the signal/noise ratio. Other surrogate methods can be used, including the coupling of an activity assay to KTGS, or thermal shift assay. ${ }^{19,43}$ Several detection methods can even be used in parallel to confirm the formation of the KTGS ligands independently. ${ }^{19}$ Unlike in FBDD, X-ray crystallography is not used as a generic method to detect ligand formation in KTGS. In the past however, X-ray crystallography 
allowed to discover unexpected ligands, like compound-substrate KTGS adducts for example.28 Thermodynamic and kinetic studies can also be performed to optimize KTGS experimental conditions before setting up larger experiments. For example Rademan et al. measured the rate of the catalyzed amidation by a kinetic enzymatic assay to select the best reagents.24 To select the best time range for the KTGS experiment, they analyzed the autoinhibition of the protein as a function of the ligand formation by HPLC-MS. Finally, the proteinligand complex was crystallized and its analysis evidenced a pattern of $\mathrm{H}$-bonds between the protein and the reactive fragments, resulting in their activation. This evidenced how the binding mode of the reactive fragments impacts the mechanism of the protein-templated reaction.

\section{In cellulo KTGS}

So far, one of the limitations of KTGS is the need for high concentration, and therefore high quantities, of purified proteins. Though this drawback is attenuated by the use of multicomponent formats and may be overcome in the future by the generalization of microfluidics ${ }^{45}$, it is partly responsible for the limited number of examples reported in the literature. Another consequence is that KTGS has also been only described for soluble protein targets. Hopefully, recently reported KTGS experiments performed directly in cells prove that it is possible to avoid target purification and to possibly expand the scope of KTGS to nonsoluble proteins. Disney et al. used in cellulo in situ-click chemistry to synthesize an inhibitor that rescued defect splicing in the myotonic dystrophy disease. ${ }^{36}$ Sellstedt et al, described the KTGS of a carbonic anhydrase II (CAII) inhibitor directly on cells (Figure 11A). ${ }^{46}$ Andrade et al. published the first ribosome-templated in cellulo in situ-click chemistry for the identification of solithromycin 1,4-triazole analogues, which activity was measured on a solithromycin resistant S.aureus stain (Figure 11B). ${ }^{37}$ In 2015, Ohkanda et al., disclosed a diterpene-peptide conjugate that was templated in cells by protein 14-3-3 via a stable oxime formation. ${ }^{47}$

This in cellulo KTGS format provides a huge advantage over KTGS using a purified target, specifically in the infectious diseases or oncology areas where the isolation of each single target protein mutant can require tremendous efforts. In the very near future, the in 
cellulo format will place KTGS as a target-engagement methodology by comparing KTGS outputs on wt-cells and ko-cells, or cells with/without a ligand.

Sellstedt et a/ 2018

A
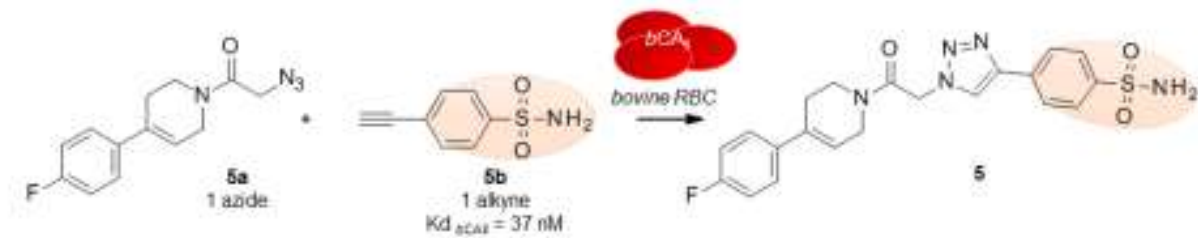

Andrade et al 2018

B
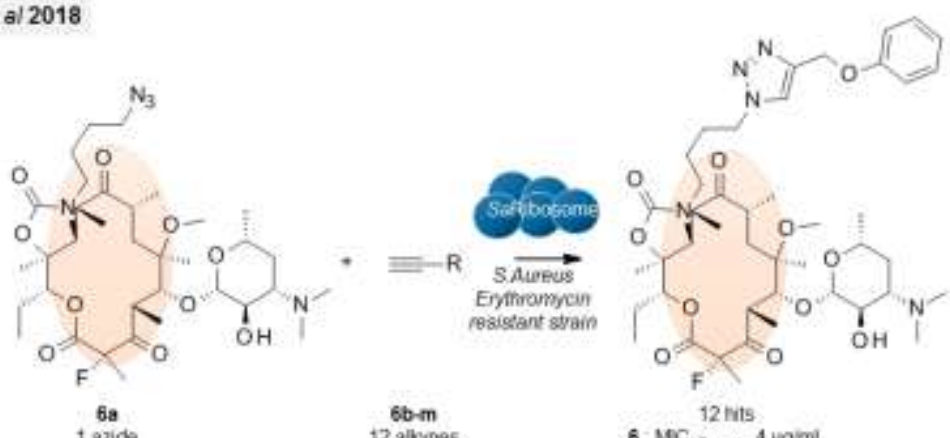

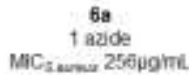

$6 b-m$
12 alknnes

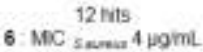

Figure 11 : Examples of in cellulo KTGS. ${ }^{a}$

a (A) Identification of templated bCAll ligand in red blood cells ${ }^{46}$; (B) Identification of a ribosome ligand in a S.aureus strain resistant to erythromycin ${ }^{37}$; Key pharmacophores are highlighted in salmon.

\section{CASE STUDIES}

\section{Recent in vitro examples.}

Inhibitors of endothiapepsin were recently obtained by Hirsch et al (Figure 12). ${ }^{39}$ They derive from hydrazide (7a) and an S-alkylated isothiourea (7b), that were previously identified by screening of a library of fragments that did not all comply the Ro3 criteria ( $39 \%$ Ro3, $61 \%$ non Ro3). ${ }^{11}$ By superimposing the X-ray structures of endothiapepsin-fragments complexes, and by linking fragments, authors modeled a potential triazole ligand. Four alkynes and nine azides were selected to perform an in situ click reaction that produced 4 hits $(5.5 \%$ hit rate, out of 72 possible anti- or syn-1,2,3 triazoles). The identified ligands were shown to display anticonfiguration as predicted by earlier molecular modeling studies. This work produced a nonclassical inhibitor of an aspartyl protease. Docking of ligand 7 in endothiapepsin showed that 1,2,3-triazole ring are in close proximity to the catalytic dyad (Asp35, Asp219). These residues are thought to change the triazole pKa value and promote protonation of the heterocycle and further hydrogen bond with Asp35. ${ }^{39}$ 


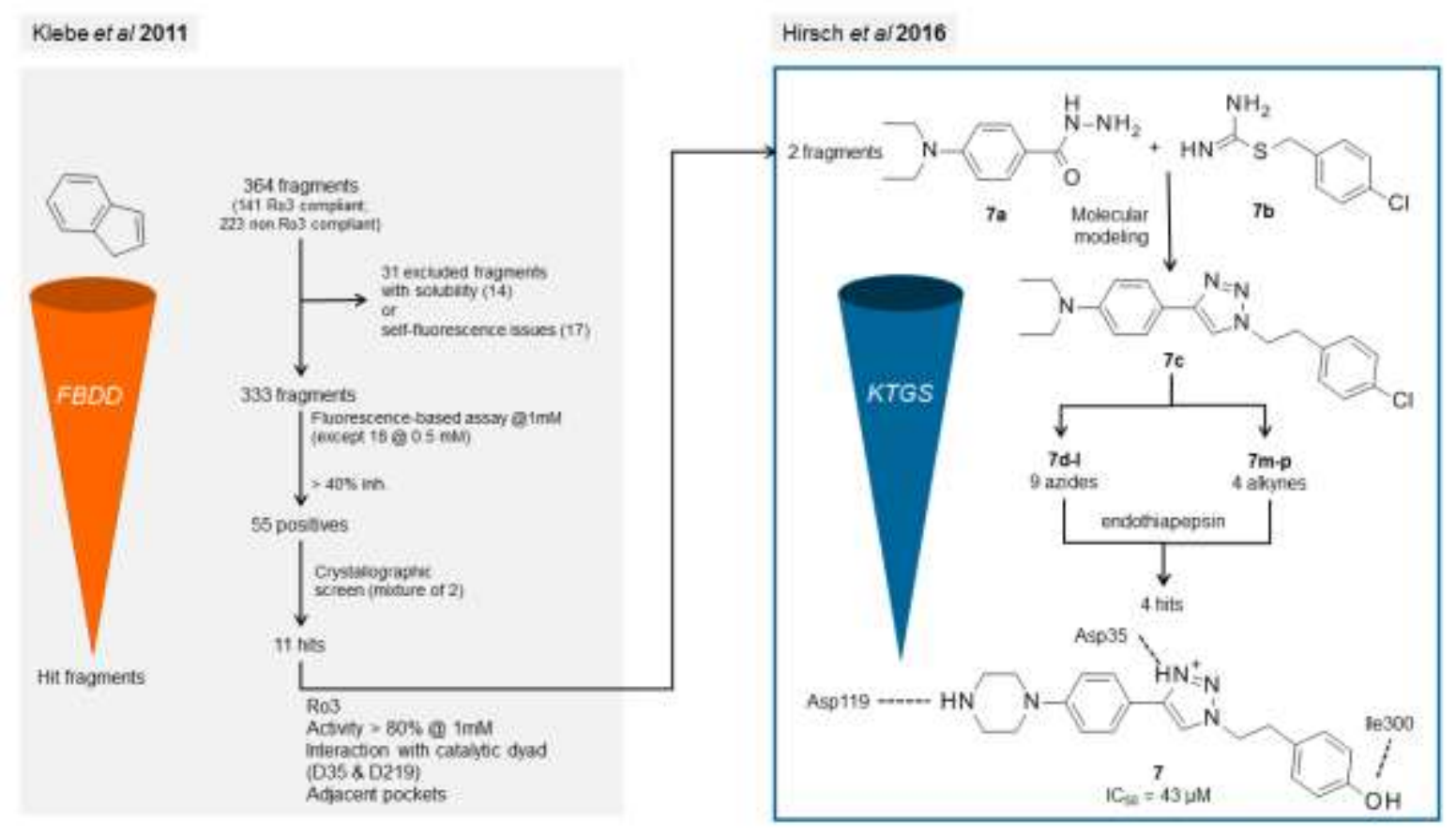

Figure 12 : Combination of non Ro3 fragment-based \& KTGS linking. ${ }^{a}$

a FBDD identified fragments that bound the catalytic dyad of the aspartyl protease endothiapepsin. Azides and alkynes were derived from these fragments and KTGS allowed the identification of 4 ligands.

A recent paper by Kwarcinski et al., disclosed the discovery of bivalent c-SRC inhibitors via a thiol-acrylamide KTGS. ${ }^{43}$ The initial warhead-bearing reagent $8 \mathbf{a}$ is an aminopyrazole derivative (Figure 13A). To perform the KTGS, an enzyme where three cysteines were mutated into serines (3M-c-SRC: C277S, C483S, C496S), was produced and used to avoid side reactions of acrylamides derivatives with the enzyme. The KTGS was performed as a binary experiment between one thiol $\mathbf{8 b}$ and 110 acrylamides (Figure 13B) and provided 4 ligands (3.6\% hit rate). Best final adduct $\mathbf{8}$ was 5 -log more potent than the acrylamide precursor $\mathbf{8 c}$ (Figure $13 \mathrm{C}, \mathrm{Ki}=160 \mu \mathrm{M})$ or thiol precursor $\mathbf{8 b}(\mathrm{Ki}=200 \mu \mathrm{M})$. Adduct 8 displays a higher ligand-efficiency than $\mathbf{8 a}$, and it is selective towards a panel of kinases thanks to the allosteric moiety brought by the acrylamide precursor. This allows 8 to bind both at the ATP binding site (hinge region) and to the P-loop, explaining selectivity (Figure 13D). ${ }^{43,48} \mathrm{KTGS}$ was successful to identify potent inhibitors of s-SRC kinase while all previously identified P-loop binding inhibitors had been discovered by structure-based drug design. 
A

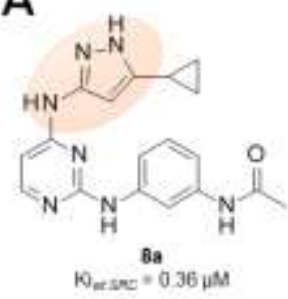

C

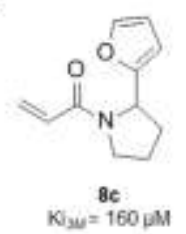

B

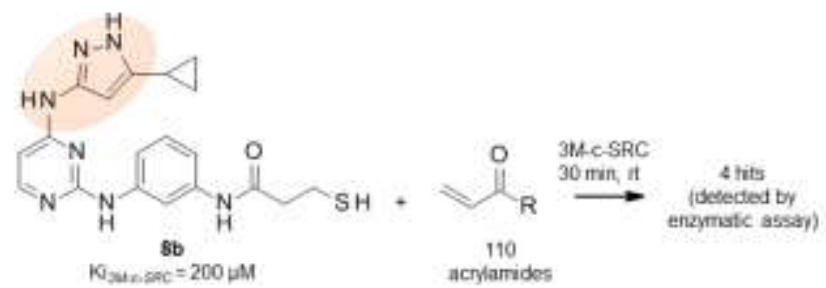

D

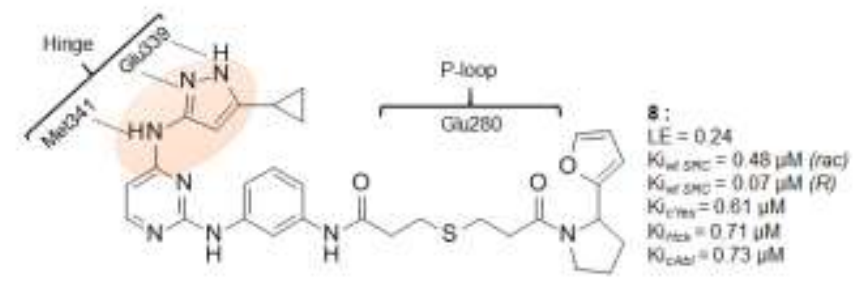

Figure 13 : Discovery of C-SRC inhibitors. ${ }^{\mathrm{a}}$

a (A) Promiscuous kinase inhibitor $\mathbf{8 a}$ that inspired the warhead-bearing reagent $\mathbf{8 b}$; (B) Protein templated reaction by kinase 3M-c-SRC protein, mutated on cysteines to avoid undesired side reactions with the pool of acrylamides; (C) activities of precursor $\mathbf{8 c}$; (D) putative binding mode of final hit $\mathbf{8}$, that interacts with the hinge reagion through the aminopyrazole group and with the P-loop of SRC via the furanylpyrrolidine group. Aminopyrazole pharmacophore is highlighted in salmon. wt: c-Src wild type; 3M-c-SRC: mutated c-Src (C277S, C483S, C496S).

KTGS was also used for the discovery of covalent inhibitors of $3 \mathrm{C}$ cysteine protease of the enterovirus $A 71 .{ }^{38}$ For this, authors used the bifunctional reagent $9 \mathrm{a}$ that bears Michael acceptor group as the covalent warhead to tether it to the catalytic Cys141, and a ketoaldehyde group to further react with 28 different ketones via aldol KTGS. Two pyridine-based covalent irreversible inhibitors were then detected by mass-spectrometry analysis of the whole protein (Figure 14A). Binding analysis of templated ligand $\mathbf{9 b}$, showed that its pyridine group fits in the P3 pocket of the enzyme (Figure 14B). Further replacement of the $\alpha, \beta$-unsaturated ester of $\mathbf{9 b}$ by an aldehyde provided a sub-micromolar reversible inhibitor $9\left(\mathrm{IC}_{50}=0.15 \mu \mathrm{M}\right)$, ten-fold more potent than rupintrivir, a rhinovirus 3C-protease inhibitor under development for the treatment of rhinovirus infections, or twice more potent than reference inhibitor NK1.8k $\left(\mathrm{IC}_{50}=0.32 \mu \mathrm{M}\right){ }^{49}$ 


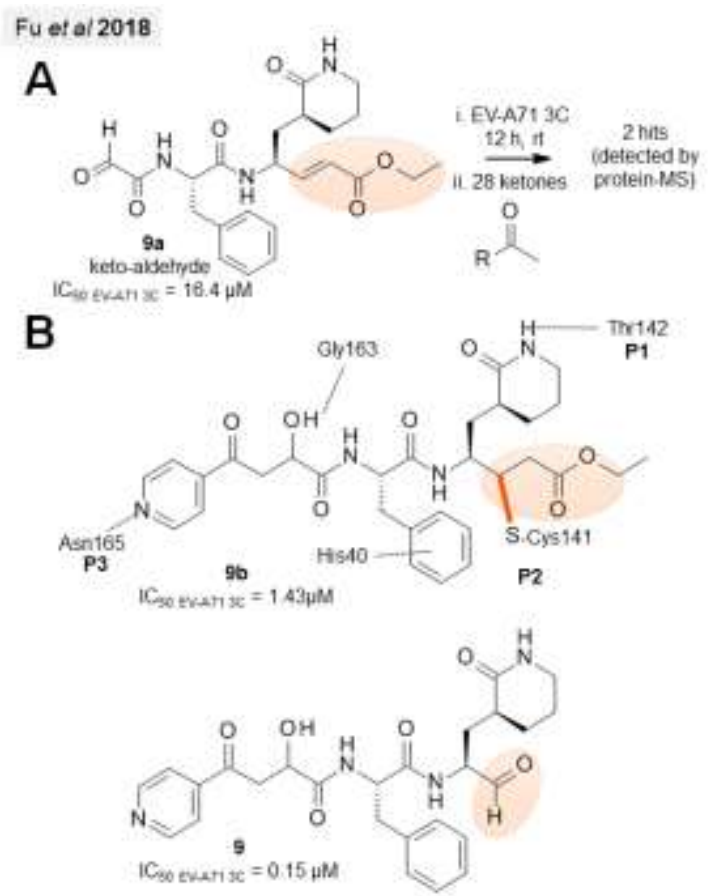

Figure 14 : Discovery of enteroviral protease inhibitors. ${ }^{a}$

a $(A)$ The ligation of precursor $9 a$ reacts with Cys 141 of EV-A71 3Cpro via its Michael acceptor group. Then the aldol KTGS between $\alpha$-ketoaldehyde moiety and ketones yields to covalent ligands detected by mass spectrometry of the protein. (B) Binding of best ligand $9 \mathbf{b}$ (orange bond: covalent bond with Cys 141) and optimized reversible analogue 9 , in which the $\alpha-\beta$, unsaturated ester has been replaced by an aldehyde. Electrophilic warhead is highlighted in salmon.

Ribosome is the biological target of ketolides like solithromycin and telithromycin (Figure 15A). A recent report describes the obtention of analogues of solithromycin using KTGS. ${ }^{50}$ This fluoroketolide displays a substituted triazole that binds a third region in the bacterial ribosome as compared to other ketolides. The multicomponent experiment allowed a rapid screening of analogues. Both syn- and anti-triazoles were detected and compound 10 displayed similar therapeutic activity as solithromycin (Figure 15B). 


\section{A}

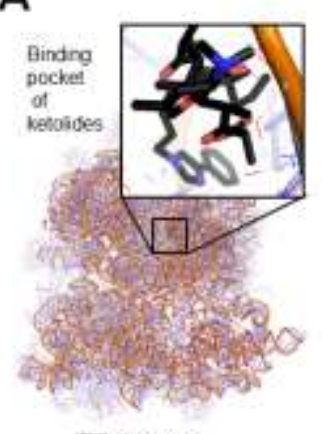

zos nbosome

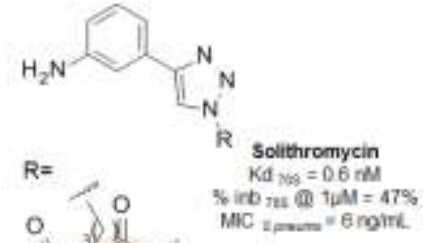

B

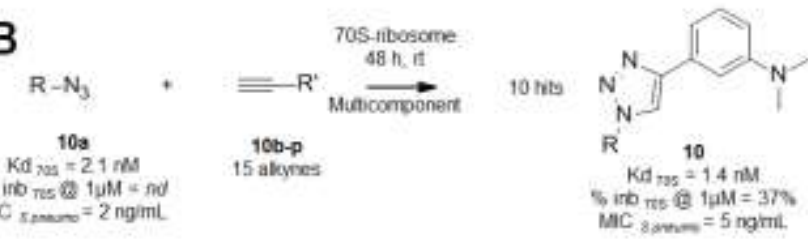

Figure 15 : Optimization of macrolides. ${ }^{a}$

a (A) Binding pocket of telithromycin within ribosome 70 S (from PDB 4v7z) and structure, binding affinity and activity of solithromycin; (B) 15-multicomponent KTGS between azide 10a and 10 diverse alkynes and structure of inhibitor 10 identified by KTGS with similar activities than solithromycin. The warhead is highlighted in salmon.

Paparella et al published the discovery of a fluorescent probe of the bacterial biotin protein ligase (BPL). ${ }^{34}$ The KTGS experiment allowed to select between two fluorescent moieties 2-[7-(dimethylamino)-2-oxo-2H-chromen-4-yl]acetamide (DMACA) versus 4-chloro7-nitro-1,2,3-benzoxadiazole (NBD), borne by the azide precursor. The KTGS was performed in parallel with BPL from 4 different bacterial strains (Figure 16). Ligand 11 was then used in bacteria and showed different distribution in E.coli and S.aureus. Indeed, while it labeled the cytoplasm of S.aureus, it only distributed at the membrane in E.coli. This explains why 1,4triazole inhibitors of BPL are permeable and active on S.aureus strains while they fail to penetrate E.coli and are thus inactive against it.

Paparella et a/ 2018

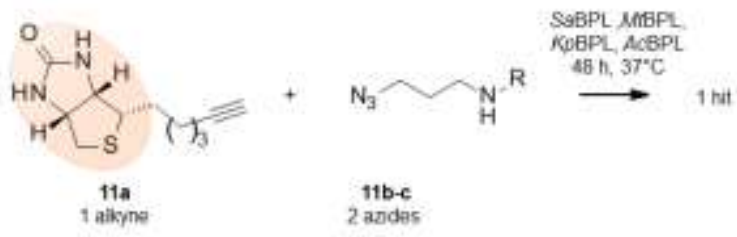

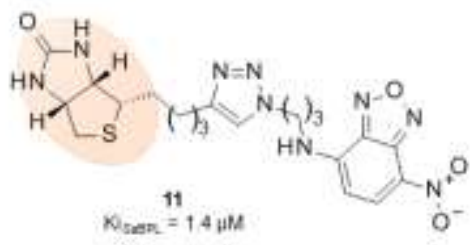

Figure 16 : Fluorescent probes of BPL. ${ }^{a}$

${ }^{a}$ KTGS probe synthesis via various Biotein Protein Ligases (PBL) and structure of the fluorescent probe 11. Biotin warhead is highlighted in salmon. 
The transfer of $\mathrm{N}$-acetylglucosamine from UDP-N-acetylglucosamine (UDP-GlcNAc) to proteins is ensured by the enzyme O-GlcNAc transferase (OGT). These post-translational events are key in the control of pathways that can be disregulated in diseases such as metabolic diseases. Wang et al. disclosed an original "mixed" tethered / multicomponent KTGS strategy to discover inhibitors of this enzyme that were further used to explore the regulation of $\mathrm{N}$-acetylglucosamination in cells (Figure 17A). ${ }^{25} \mathrm{~A}$ bifunctional covalent warhead-bearing reagent (iodo-acetamide azide) 12a reacted first with OGT cysteines, then mixtures of alkynes were added (Figure 17A). Fourteen hits were identified. Then, the electrophilic group of best compound $\mathbf{1 2 b}$ was removed to provide the cell-permeable, non-cytotoxic, non-competitive, non-covalent inhibitor 12 (Figure 17B). Docking experiments allowed to propose a binding mode within the enzyme (Figure 17B). 12 was tested in COS-7 cells and showed to reduce the quantity of O-GlcNAcase, a protein that catalyzes the opposite reaction and whose quantities are reduced when OGT is inhibited.

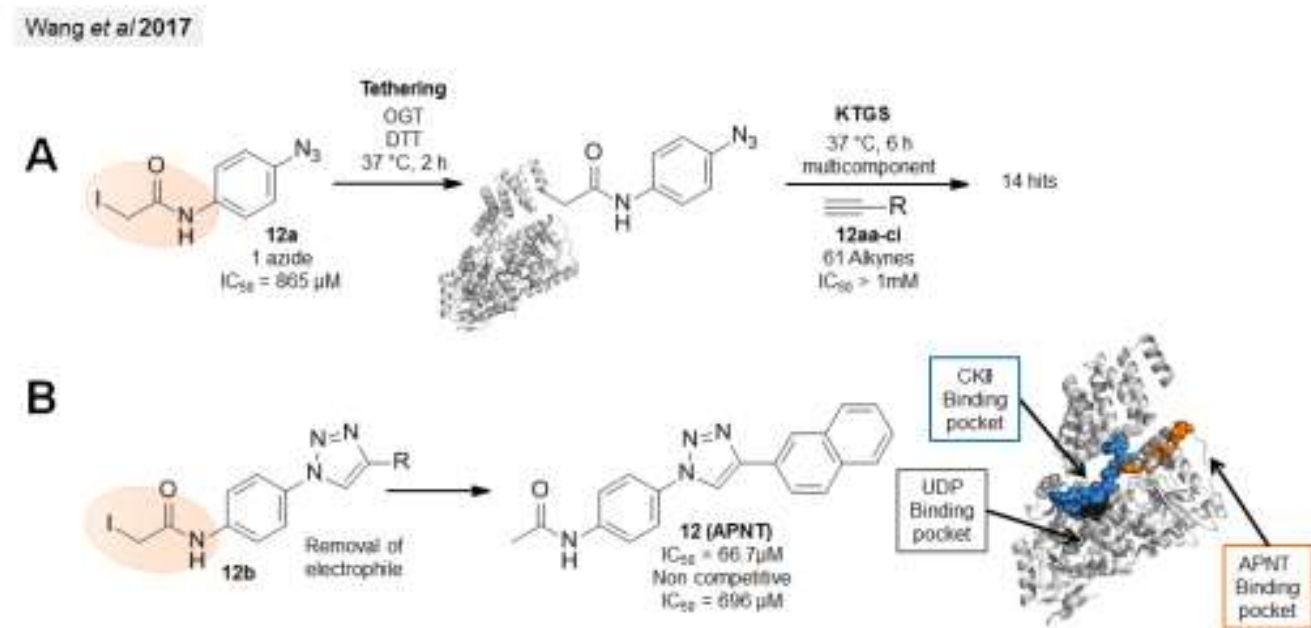

Figure 17 : Cell-permeable O-GIcNAc transferase inhibitors. ${ }^{a}$

a (A) Tethering-in situ click chemistry (TISCC) to discover inhibitors of O-GlcNAc transferase (OGT): iodinated azide reacts covalently with OGT protein (Tethering), then templated reaction of triazole from a pool of 61 azides allowed the identification of 14 hits ; (B) Non-competitive inhibitor 12 derived from covalent KTGS ligand $12 b$ by removal of iodo-acetamide group. Putative binding site of 12 in OGT (grey, PDB code : 3PE4), peptidic inhibitor in blue, UDP in black, APNT binding residues in orange. Electrophilic warhead is highlighted in salmon.

\section{Recent in vivo examples.}

In 2013, Kolb et al disclosed a PET-scan probe targeting carbonic anhydrase II (CAII), from a ${ }^{19} \mathrm{~F}$-containing fragment library and the 4-ethynyl benzenesulfonamide $(\mathrm{Kd}=37 \mathrm{nM})$ as a warhead binding the catalytic $\mathrm{Zn}^{2+}$ ion (Figure 18A). ${ }^{51}$ The probe $\left[{ }^{18} \mathrm{~F}\right]-13$ (Figure 18B) was 
then synthesized from the nitro-containing, dimethoxytrityl-sulfonamide protected analogue and then converted to the labeled derivative with $\left[{ }^{18} \mathrm{~F}\right]$-fluoride, in the presence of crown-ether in acetonitrile, followed by deprotection and HPLC purification. The probe was directly administered to mice (Figure 18C) and its distribution was monitored over 1 hour. The probe distributed in some organs (heart, kidney), and tissues (blood) consistently with the expression and distribution of CAll. Authors checked that the probe mainly bind red blood cells (RBC) rather than plasma (90/10 ratio) in blood. No defluorination during the experiment was observed.

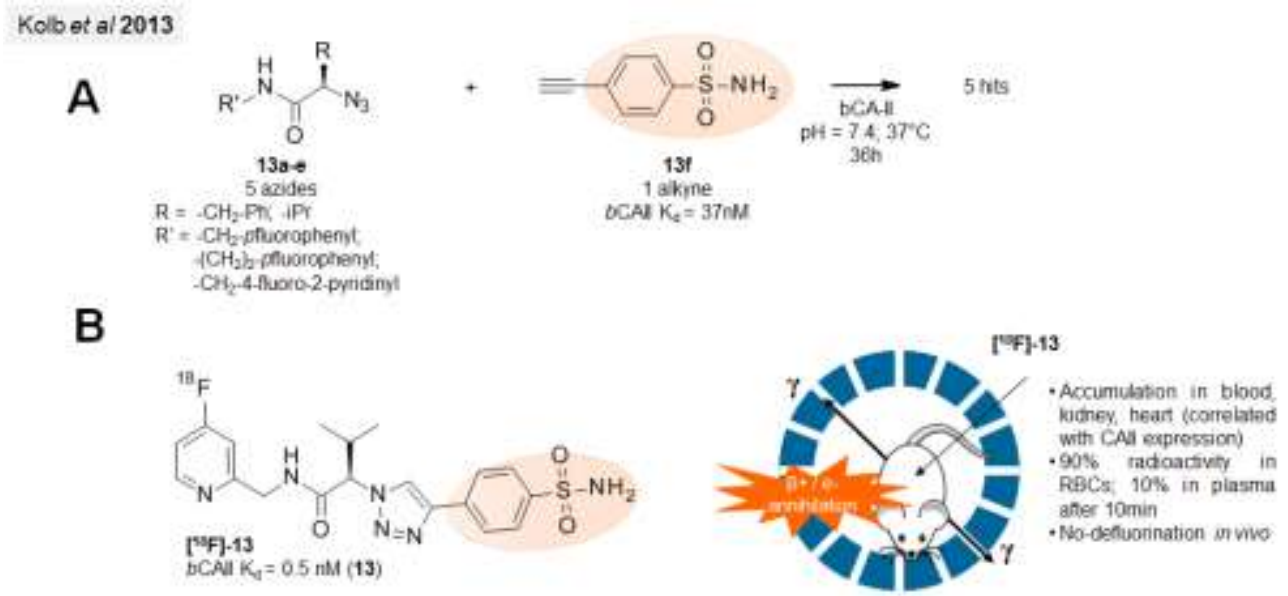

Figure 18 : Discovery of a PET-scan probe for carbonic anhydrase II. ${ }^{a}$

${ }^{a}$ (A) KTGS of bCAll ligands from azides and benzylsulfonamide warhead. (B) structure of probe $\left[{ }^{18} \mathrm{~F}\right]-13$, direct ${ }^{18} \mathrm{~F}$ labeled analogue of a KTGS-hit 13 and its stability and distribution measured by PET scan. RBC: red blood cells.

\section{Deprez-Poulain et al 2015}

A
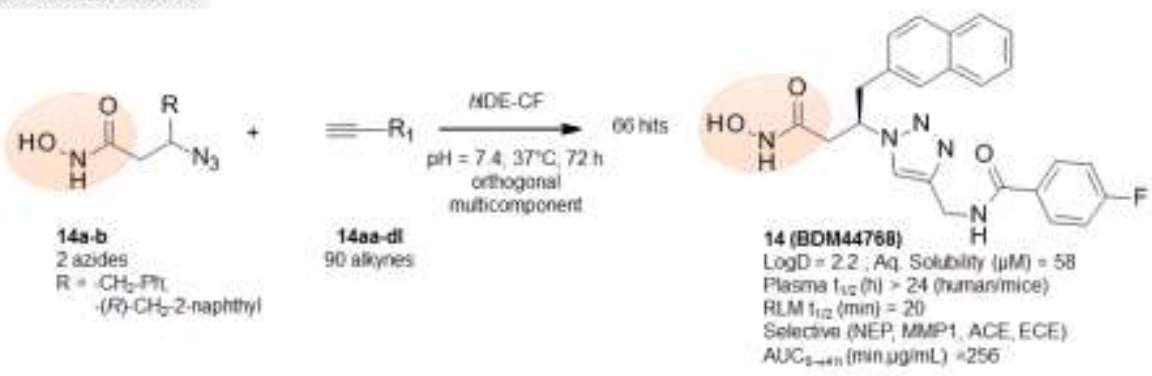

B

C
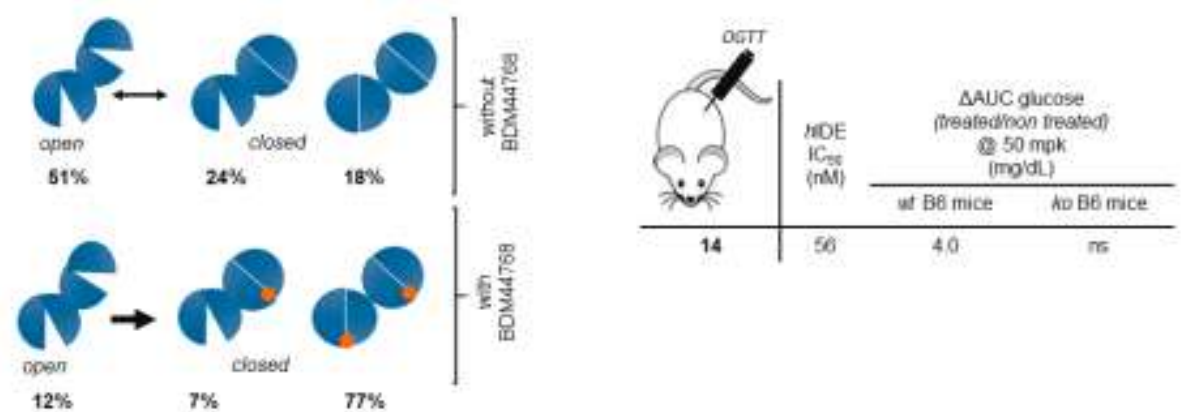


\section{Figure 19 : Inhibitor of Insulin-degrading enzyme. ${ }^{a}$}

a (A) KTGS by hIDE-CF using an orthogonal multicomponent strategy, from 2 azides bearing an hydroxamic acid warhead and 90 diverse alkynes; Best hIDE inhibitor 14 (BDM44768) and its ADME and selectivity properties; (B) 14 (orange sphere), shifts open/closed equilibrium of hIDE towards closed conformations, measured by Small Angle X-ray scattering (SAXS); (C) Inhibition of hIDE by 14 and effect on in vivo glucose tolerance as the ratio of glucose AUC treated/non treated. The effect is IDE dependent ( $w t$ vs IDE ko). ns: non significative; CF: cysteine-free.

In 2015, we disclosed the first insulin-degrading enzyme inhibitor discovered by KTGS which was active in vivo. ${ }^{31}$ The experiment was run as an orthogonal multicomponent set up (Figure 19A). Best compound, 14 (BDM4478), displayed good ADME properties (Figure 19A). In particular, its hydroxamic function was shown to be stable towards esterase hydrolysis. ${ }^{52}$ It interacts with the zinc ion of Insulin degrading enzyme and shifts the equilibrium towards the close conformation of IDE (Figure 19B). In an in vivo glucose tolerance test, 14 enhances glucose intolerance in an IDE-dependent manner (Figure 19C). This compound allowed us to invalidate this enzyme as a type-2-diabetes target.

Using KTGS, Kolb et al also identified several inhibitors of $h$ COX-2 that display the benzenesulfonamide warhead from celecoxib (Figure 20A). Inhibitor 15 was further labeled with ${ }^{18} \mathrm{~F}$ to be used in vivo as a PET-scan probe. ${ }^{53}$ In 2017 , Wuest et al designed a multicomponent experiment to identify 2 potent 1,4-triazole inhibitors of COX-2. The experiment involved 4 azides 16a-d and 11 alkynes $16 \mathrm{e}-\mathrm{o}$ to give potentially 88 adducts (either 1,4 or 1,5 triazoles). Two hits (16 and $\left.\mathbf{1 6}^{\prime}\right)$ were identified (2.2\% hit rate), with an $\mathrm{IC}_{50}$ of 50 and $90 \mathrm{nM}$ respectively, in the range of the $\mathrm{IC}_{50}$ of celecoxib $(70 \mathrm{nM})$. The compounds were also active in a cellular assay on HCA-7 colorectal cancer cell lines. 16 and 16' were tested in an in vivo model of inflammation (carrageenan-induced rat paw edema) and were shown to be more potent than celecoxib in that model. 


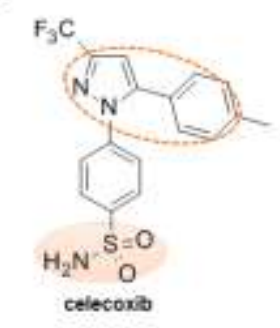

Wuest et a/ 2017

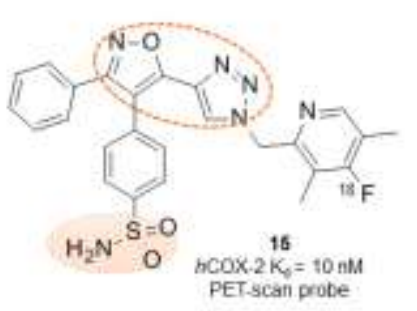

B

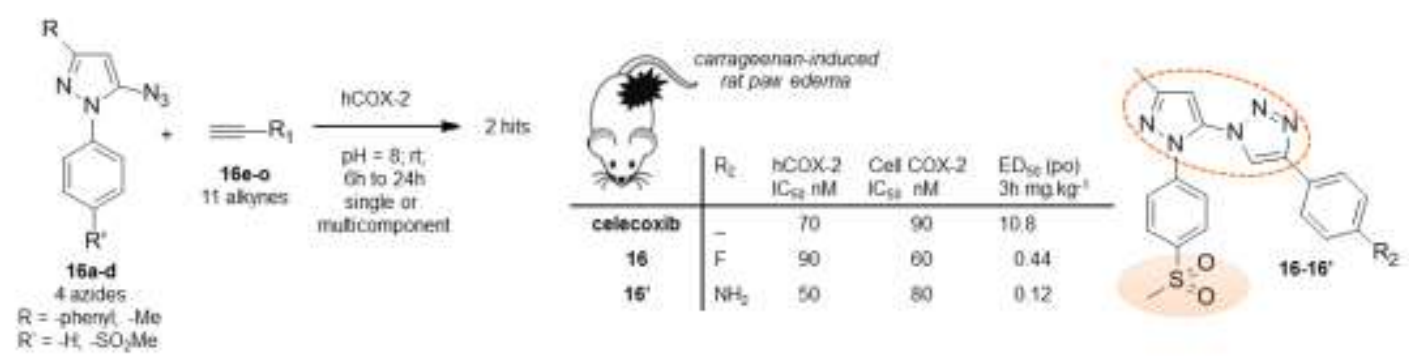

Figure 20 : hCOX-2 inhibitors in vivo. ${ }^{\text {a }}$

a (A) Structure of the drug celecoxib; and of the PET-scan probe 15, labeled analogue of a KTGS ligand inspired by celecoxib; (B) KTGS of COX-2 inhibitors from celecoxib -inspired azides 16a-d and 11 alkynes 16e-o and structures and activities of KTGS ligands 16-16' in an inflammation rodent model. Biaryle pharmacophore is circled with an orange dotted line; sulfonamide or sulfoxide warhead is highlighted in salmon.

STAT5b inhibitors prevent dimerization of phosphorylated STAT5b, thus inhibiting transcription of genes implicated in cancer cells proliferation and survival (Figure 21A). Rademann et al combined fragment screening and a Mannich-based KTGS (Figure 5B) to discover new STAT5b inhibitors from the 1,2,5-oxadiazole warhead-bearing reagent 4a (Figure 21B). ${ }^{19}$ This is the first series of protein-templated inhibitors of STAT5b. Several hits $(\mathbf{4 x - z})$ displayed micromolar-range inhibition of a phosphopeptide-STAT5b interaction. In particular compound $4 \mathbf{x}(1.4 \mu \mathrm{M})$ was shown to engage the target in cells $\left(\mathrm{CETSA} \Delta \mathrm{Tm}=8^{\circ} \mathrm{C}\right)$ with an in cell target occupancy concentration $\left(\mathrm{OC}_{50}\right)$ of $28 \mu \mathrm{M}$ (Figure $21 \mathrm{C}$ ) evaluated by ITDRF (Isothermal dose-response fingerprint). Its methyl ester prodrug $\mathbf{4 z}$ inhibits proliferation of cancer cells with an $\mathrm{IC}_{50}$ of $25 \mu \mathrm{M}$. A more lipophilic analogue of $\mathbf{4 x}$, compound $\mathbf{4 y}$, induces tumor regression at $200 \mathrm{mg} / \mathrm{kg}$ (Figure 21C). 
A

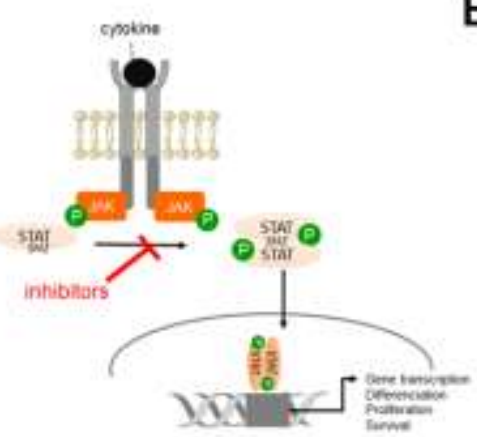

B

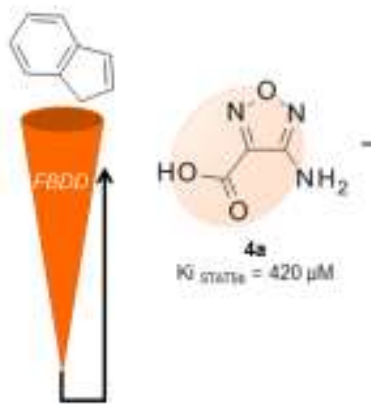

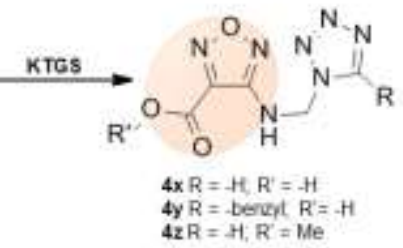

C

\begin{tabular}{|c|c|c|c|c|c|c|}
\hline & $\begin{array}{l}\text { interactioet } \\
x_{-50} \text { uth }\end{array}$ & $\begin{array}{l}\text { CETSA } \\
\text { STATSO } \\
\text { ATm "C }\end{array}$ & $\begin{array}{l}\text { Protferation n } \\
\mathrm{K}_{\text {sn }} \text { iM }\end{array}$ & $\begin{array}{l}\text { STAT5o } \\
\text { phosphonlation to a } \\
25 \text { sul }\end{array}$ & $\begin{array}{l}\text { STATSD } \\
\text { occupancy } \\
\text { OC: }\end{array}$ & $\begin{array}{l}\text { Tumor growth } \\
\text { regression } \\
\text { Q day } 14 \\
\text { (sc) } 200 \mathrm{mg} \mathrm{kg}^{-1}\end{array}$ \\
\hline $4 x$ & 1.4 & 8 & 153 & - & 28 & - \\
\hline $4 y$ & 29 & - & 78 & $>50 \%$ & - & 205 \\
\hline $4 z$ & 37 & - & 25 & - & - & - \\
\hline
\end{tabular}

Figure 21 : Cellular and in vivo activity of STAT5b inhibitors discovered by KTGS. ${ }^{a}$

a (A) Mode of action of STAT5b inhibitors; (B) FBDD allowed the identification of a fragment 17a and subsequent KTGS gave STAT5b inhibitors $\mathbf{4 x - z}$; (C) in vitro and in vivo activities of KTGS ligands $\mathbf{1 7 b}$ - $\mathbf{d}$. ${ }^{\mathrm{b}}$ measured by fluorescence polarization assay with labeled phosphotyrosine peptide and fused maltose-binding protein(MBP)STATb-SH2; c measured in BaF3/FLT3-ITD cells; ${ }^{d}$ quantified by western-blot after $6 \mathrm{~h}$; ${ }^{\mathrm{e}}$ measured by cellular thermal shift assay (CETSA) -ITDRF; ${ }^{\dagger}$ measured in BaF3/FTL3-ITD xenograft tumor model.

\section{RELEVANCE OF KTGS FOR MEDICINAL CHEMISTRY AND DRUG DISCOVERY}

\section{Therapeutic areas and protein targets}

Already nine different therapeutic areas are covered by the KTGS published examples (Figure 22). This emphasizes the usefulness of the strategy. Infections, cancer and CNS diseases bring together the highest number of examples. New areas were recently tackled like cardiovascular diseases.

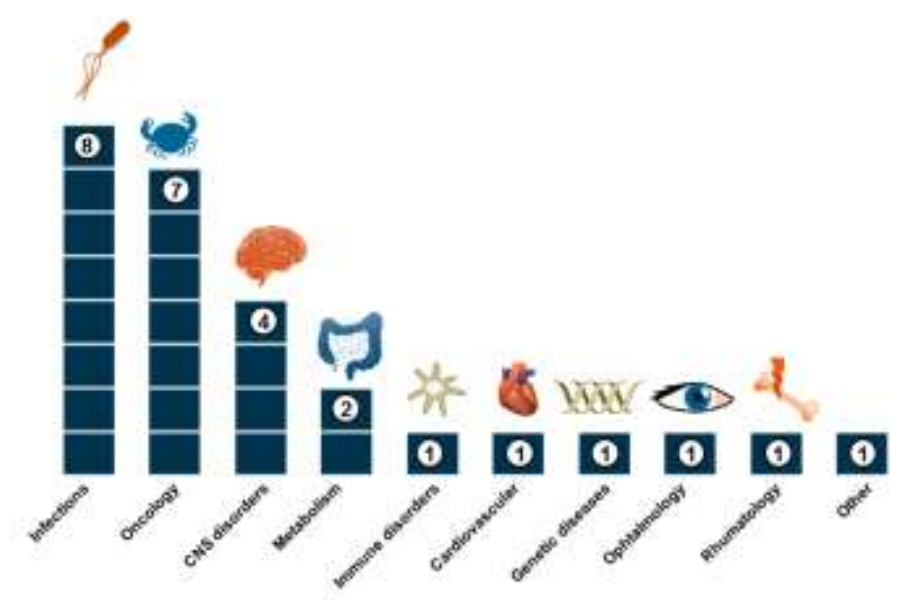

Figure 22 : KTGS experiments sorted by therapeutic areas ${ }^{a}$

a Other: endothiapepsine is a model enzyme for aspartylproteases involved in different therapeutic areas. O-GlcNAc transferase was classified in metabolic diseases for its implications in insulin resistance, diabetic complications though it is also implicated in other areas also like cardiovascular diseases or Alzheimer's disease ${ }^{25}$. 
A

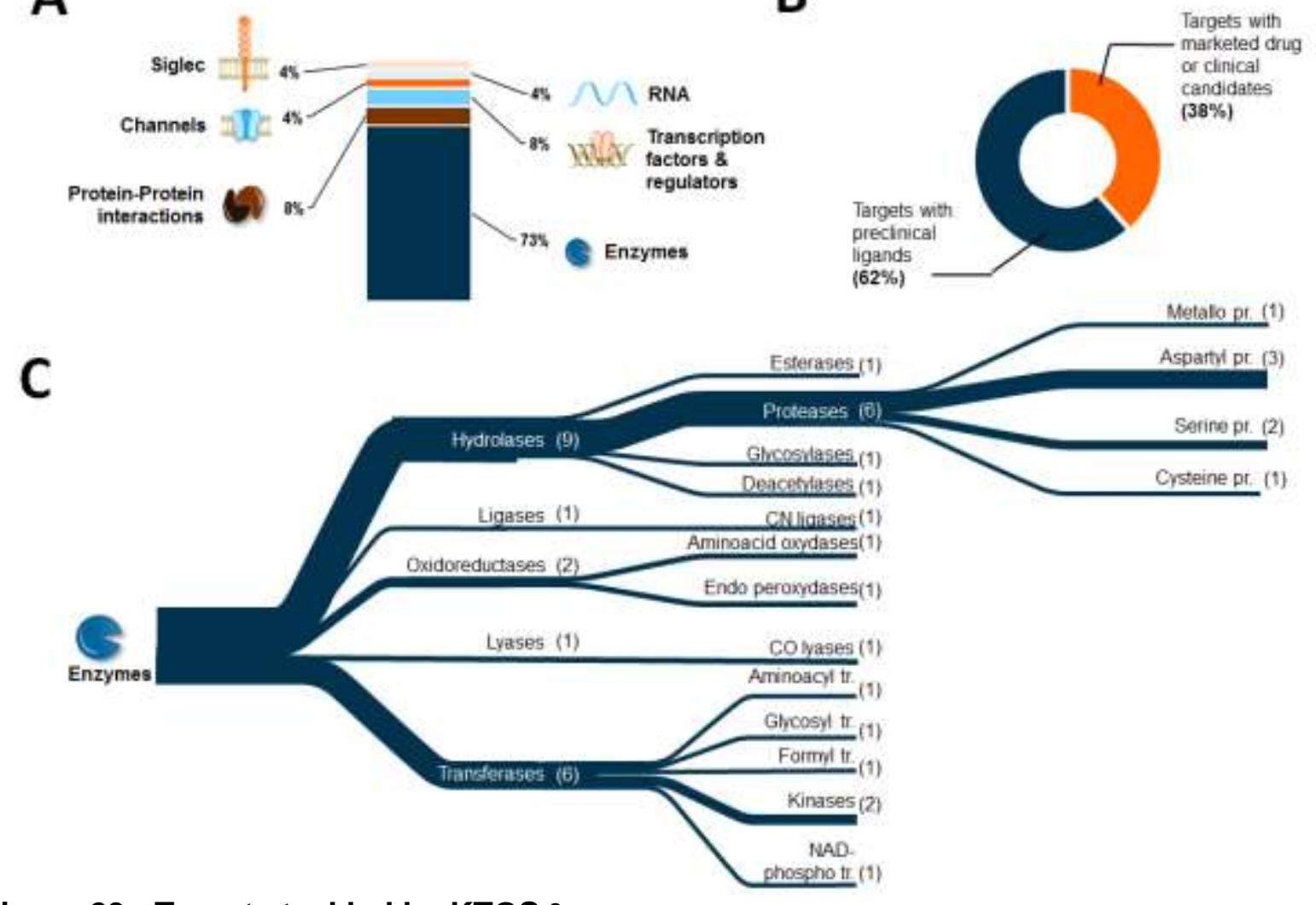

Figure 23 : Targets tackled by KTGS. ${ }^{a}$

a (A) Proportion of KTGS examples by target types. Enzymes account for more than $70 \%$ of the cases. (B) Proportion of targets with approved drug or clinical candidates. (C) Number of KTGS examples by enzyme family, showing that almost all enzymes classes can be targeted by KTGS.

KTGS has already been applied to 26 different targets from various classes (Figure 23A). $73 \%$ of these targets are enzymes, $8 \%$ protein-protein interactions, and $8 \%$ transcriptional receptors and $4 \%$ channels (allosteric site). Among these targets, $38 \%$ have already yielded to clinical compounds or marketed drugs and $62 \%$ are either new targets or druggable targets for which some preclinical ligands have been disclosed showing that KTGS is used in very early phases of drug-discovery (Figure 23B). This shows that KTGS can be successfully applied to "old" and new targets. For enzymes, all subclasses have been explored, hydrolases and transferases being the most popular ones (Figure 23C). As mentioned above, all these targets are soluble: enzymes, protein-protein interaction partners, transcriptional receptor. The ligand-gated ion channel example reported in the literature is acetylcholine binding protein (AChBP), a soluble extracellular domain, homologue of nicotinic acetylcholine receptors (nAChRs) allosteric site that is secreted by mollusks. ${ }^{20}$ In the past years, new families of targets have been tackled by KTGS such as transferases (ribosome and 
glycosyltransferase) and the four classes of proteases: metalloproteases (IDE), serine proteases (factor Xa), aspartylproteases (endothiapepsine), cysteine proteases (enteroviral EV Cys protease)

Other preliminary disclosures, and thus not included in this study, mention KTGS experiments on new proteins to discover inhibitors of the isomerase MIF (macrophage migration inhibitory factor) in autoimmune diseases \& immuno-oncology ${ }^{54}$ ligands of $\mathrm{Mcl} 1$, for protein-protein interactions in cancer ${ }^{55}$ or multitarget inhibitors of BACE-1 and kinases in Alzheimer's disease ${ }^{56}$

\section{Protein pockets and conformation}

Hirsch and co-workers recently analyzed the geometry and the physicochemical properties of catalytic pockets of a set of 12 targets out of the 23 proteins used at the time of their paper (Table 1).22 Since no targets for which KTGS failed are reported, the « negative » control of comparison was a set randomly chosen of 20 protein targets with an approved drug. The different pockets were given a score considering their size, depth, enclosure, surface, and nature of amino acids. The parameters studied in this work showed a high variance due to the limited available data of the restricted number of reported targets successfully used in KTGS. Although, the mean depth of KTGS pockets (Table 1) is very similar to the reference set (26 vs $26.5 \AA$ ), the former contain more polar amino acids, and are slightly wider ( $1445 \AA^{3}$ vs 1338 $\AA^{3}$ ). Besides, enclosure score of the KTGS pockets (Table 1), considering simulated solvent exposure, resulted to be significantly different from the negative control $(0.114$ vs 0.077$)$. This suggests that the KTGS pockets are less deep and more open. The authors speculate that the more exposed pockets in reported KTGS examples may allow a better diffusion of reagents within this pocket and contribute to the success of the templated-reaction. Still these results might be biased by the fact that the KTGS target set contained three PPI targets (among which Bcl-XL and 14-3-3 protein) that are typically larger and very shallow. However, no particular physico-chemical property let distinguish KTGS pockets from the reference set, suggesting that KTGS can have a large target scope, regardless the geometry and properties of the active 
sites. The authors also showed that the parameters measured for KTGS pockets qualify them as "druggable" pockets as defined by Vollkamer et al. ${ }^{57}$

Supplementary Table 1 gathers all the binding pockets of KTGS ligands for each example fully disclosed in the literature. For enzymes, almost all ligands target the catalytic site. This is not surprising because warhead usually target specific features of this site. Still a few example report new binding pockets and/or new conformations. KTGS is indeed complementary to X-ray crystallography, NMR of proteins and molecular modeling as it can evidence unknown and less abundant protein conformations. For example, a new "open-gate" conformation, due to an unexpected flip of two phenylalanine residues, was discovered during the KTGS formation of the triazole ligand in TbEthR. It unveiled a new pocket within the protein. This new conformation impacted the activity of this transcriptional repressor. ${ }^{18}$ Inhibitor 14 of $h \mathrm{IDE}$, interacts with the catalytic site formed by both IDE-N and IDE-C and impacts the dynamics of the door subdomain of the enzyme thus impacting its catalytic activity. ${ }^{58}$ Bourne et al showed recently that the binding of one KTGS warhead-bearing reagent to AChE induces the motion of a histidine residue in the catalytic site, along with an unprecedented motion of a tyrosine residue in the gorge of the protein. These conformational changes within the protein result in the formation of the preferred KTGS 1,5-triazole ligand. ${ }^{59}$

Table 1 : KTGS Pockets features ${ }^{\mathrm{a}}$.

\begin{tabular}{|c|c|c|c|c|}
\hline & $\begin{array}{c}\text { volume } \\
\left(\AA^{3}\right)\end{array}$ & depth $(\dot{A})^{b}$ & Enclosure $^{c}$ & $\begin{array}{c}\text { fraction of apolar } \\
\text { amino acids }\end{array}$ \\
\hline KTGS pockets & 1445 & 26 & 0.114 & 0.42 \\
\hline Reference set of drug pockets & 1338 & 26.5 & 0.077 & 0.53 \\
\hline
\end{tabular}

\section{Chemical space and ligand efficiency of KTGS ligands}

The KTGS strategy offers the possibility to explore a chemical space wider than the Ro5 space. Most of the templated ligands do not observe the Lipinski-Veber rules for at least one $(74 \%)$ or two (62\%) molecular properties but significant proportions of the KTGS ligands comply the beyond the rule-of-5 (bRo5) rule, mainly around the frontier of the Ro5 chemical 
space. Therefore most of the KTGS ligands (82\%) are in the "possible to be oral » chemical space (Figure 24) as defined by Doak et al. ${ }^{60}$ For example, while only $38 \%$ of KTGS ligands fulfill the Ro5 range for MW whereas $97 \%$ of them fulfill the Doak's MW range (ie. MW $\leq 1000$ ).

A

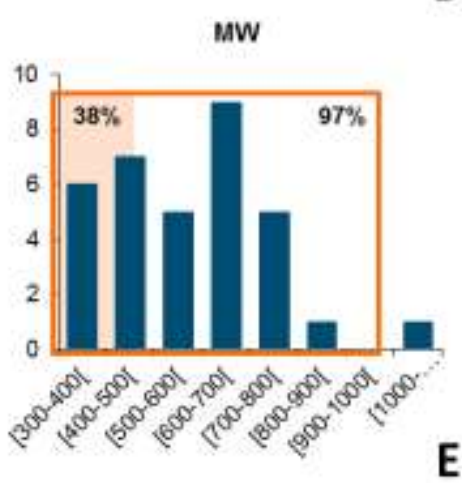

HBA

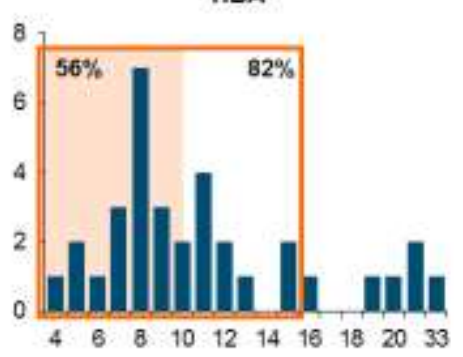

B

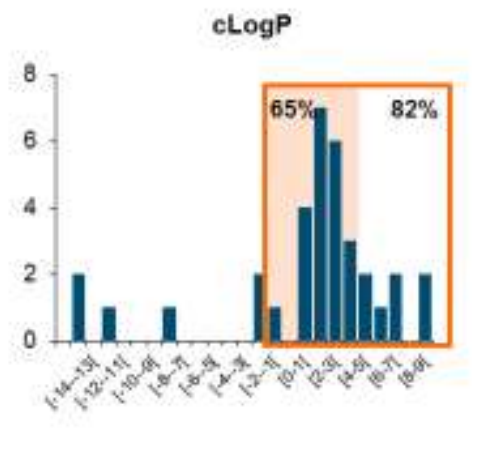

HBD

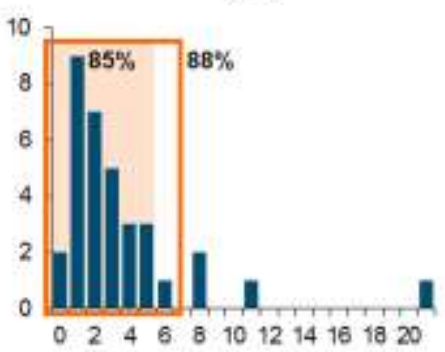

C

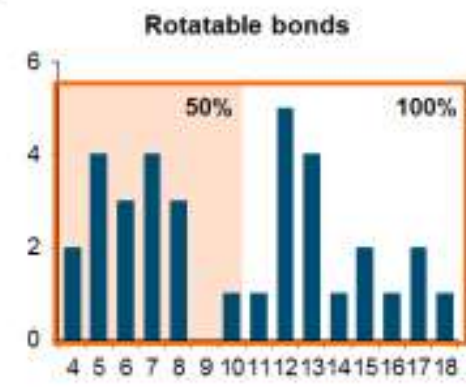

$\mathbf{F}$

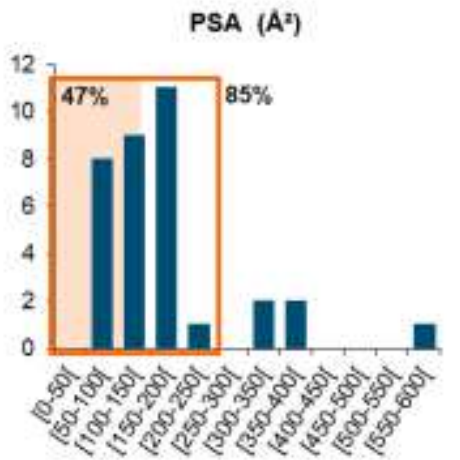

\section{Figure 24 : Structural features of KTGS ligands. ${ }^{a}$}

a Distribution of the (A) MW, (B) cLogP, (C) number of rotatable bonds, (D) number of HBA, (E) number of HBD, (F) PSA for KTGS ligands (1 example selected/KTGS experiment). 38\% of KTGS ligand fulfill the classical druglike rules for all parameters but $82 \%$ fit within the Doak's possibly oral chemical space (MW $\leq 1000 ;-2 \leq$ cLogP $\leq$ $10 ; \mathrm{HBD} \leq 6$; HBA $\leq 15$; PSA $\leq 250 \AA^{2}$; rotatable bonds $\leq 20$ ). In salmon filled boxes: rule of $5 /$ Veber compliant compounds; In orange open boxes: "possibly oral chemical space".

The physico-chemical and structural parameters of KTGS ligands were plotted against the ones of FDA approved drugs (Suppl. Fig. 1 \& 2). Except for the four KTGS ligands that contain sugar moieties, all compounds distribute well within the chemical space defined by FDA approved drugs (Suppl. Fig. 1). A one-to-one comparison for each parameter (Suppl. Fig. 2) via box plots shows that KTGS ligands display a larger size (MW), are more flexible (rotatable bonds) and more polar (PSA) compared to the FDA approved drugs. Regarding rotatable bonds, a high variance is observed for KTGS ligands and the histogram distribution shows two populations of ligands: one centered on $\mathrm{nRot}=6-7$ and one centered on $\mathrm{nRot}=12$ 13 (Suppl. Fig. 2). As stated earlier by Doak et al for oral drugs, the HBA parameter is largely 
responsible for the increased PSA of high-molecular weight compounds. ${ }^{60}$ As well, the fact that KTGS ligands show higher PSA values in comparison with FDA drugs, could be correlated to the increase of HBA number. Out of the KTGS ligands, $72 \%$ have a lipophilic ligand efficiency (LLE) above 3 (Figure 25A), with a mean of 5.0 and a median of 4.9. This qualifies KTGS ligands as good starting points for medicinal chemistry efforts. Importantly, KTGS itself increases LLE (from warhead-bearing reagent to final ligand) (Figure 25B) up to 6 units, making it a valuable tool for ligand optimization.

A

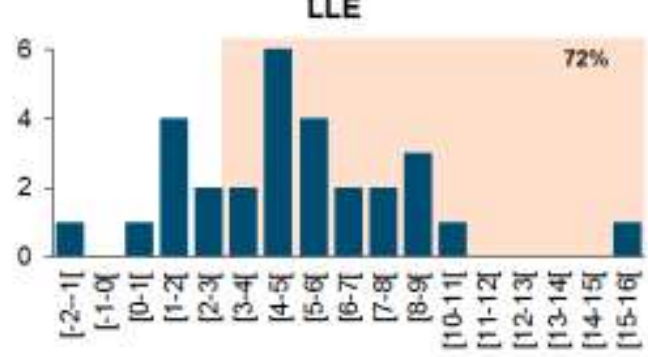

B

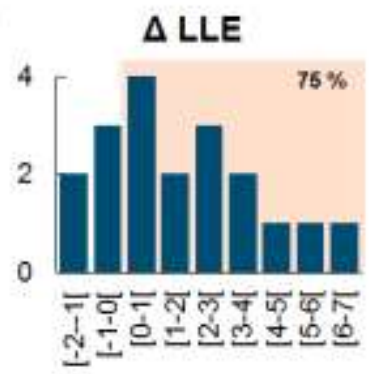

Figure 25 : LLE distribution and LLE changes. ${ }^{a}$

a Distribution of Lipophilic Ligand Efficiency LLE for reported KTGS ligands (A) and change in LLE during KTGS process $(B)$. Only for pairs where both the warhead-bearing reagent and the KTGS ligand had numerical values $(\mathrm{N}=36)$. A: $72 \%$ of KTGS ligands have a LLE > 3 (in salmon) (threshold for medchem starting points) and $25 \%$ a LLE $>6$ (threshold for quality drug candidates). B: $75 \%$ of KTGS allow an increase in LLE ( $\Delta$ LLE $>0$ in salmon).

\section{CONCLUSIONS}

Kinetic Target guided Synthesis has now reached its maturity as a hit discovery/optimization strategy with more than 50 reported examples. It has allowed the discovery of ligands for 26 different targets within 10 different therapeutic areas. The analysis of KTGS ligand properties has shown that it can be widely used in medicinal chemistry programs as it allows to access ligands with adequate properties for optimization: $72 \%$ of them display a LLE above $>3$ and $82 \%$ comply with the "possible to be oral chemical space" properties.

KTGS scope is increasing as it was shown to be successful for both targets with an approved drug or clinical candidate, or with new or more difficult targets. It affords hit discovery as well as the generation of alternative starting points, or the exploration of unknown protein 
conformations. ${ }^{18,31}$ Target types range from enzymes to more "recent" RNA or protein-protein interactions. Binding sites span from catalytic pockets, allosteric sites, protein-interaction sites, to phosphate sites. In addition, already 4 examples of KTGS-discovered ligands have been tested in vivo for imaging or therapeutic intervention. The synthetic toolbox of KTGS has increased in the past couple of years to include multicomponent reactions and amidation, two favorite reactions of medicinal chemists. ${ }^{27}$ It now encompasses 9 different types of reactions. The ligands discovered by these newly developed KTGS reactions bear in their structure obvious disconnection points that facilitate their optimization.

A major current limitation of KTGS is the need for soluble protein at large amounts. As a consequence, several target types, like membrane bound receptors, have not been explored so far. Another drawback may be the need for biocompatible reagents as KTGS is performed in aqueous media. This explains why 1,3 cycloadddition from azide and alkynes is so popular as both azides and alkynes are biocompatible. Moreover, if one uses electrophile reagents like acrylamides, it may be necessary to produce a protein mutant deprived of cysteine residues to avoid side reactions. The success of KTGS greatly depends also on the type of warheadbearing reagent used to target pockets within the protein of interest. We evidenced that these warhead-bearing reagents come from either drugs, known SAR or new fragments. Finally one limitation is the detection of the templated ligand by mass-spectrometry. Still, this problem is not as critical for KTGS than for DCC where an enrichment in one compound within a dynamic library must be observed. The continuous improvement of MS sensitivity/selectivity will undoubtedly increase the usefulness and spread of KTGS in the future.

On-going opportunities for KTGS include the development of covalent inhibitors, the combination with FBDD, the in cellulo KTGS, the qualification of new MCR, detection methods and collaborative networks. Already 5\% of KTGS ligands are covalent inhibitors. Future development could make use of warhead-bearing reagents that display alternative reactive groups to fine-tune binding kinetics of such inhibitors. ${ }^{61}$ Also, $15.6 \%$ of the reported examples started from a fragment screening. With the largely used fragment screening it is expected that more examples that combine these 2 strategies will emerge. In cellulo KTGS allows now to 
explore intra-cellular targets. Theoretically this decreases also the use of large amounts of protein to be isolated and gives access to a more relevant cellular context. However, the ligand formed by a protein-templated reaction between reagents that cross cell membrane, or bacterial walls, will not be necessarily as penetrant as its precursors. This should be taken into consideration. The multicomponent and orthogonal multicomponent formats should be widely used also to decrease the protein amounts required and take the full benefit of a kinetically driven competition between large sets of reagents. Regarding chemical reactions amenable for KTGS, the development of multicomponent reactions is highly desirable in the future. In the course of the writing of this review, Van der Veken's team reported a systematic study to develop a protein-templated version of a 3-component reaction (Groebke-BlackburnBienaymé) for the discovery of urokinase inhibitors. ${ }^{62}$ Such an exhaustive study of experimental parameters should be applied to all new KTGS reactions. As well, a systematic study of the stability and integrity of the target protein should be performed to "qualify" the target for KTGS. ${ }^{5}$ In the future, the use of several detection methods in parallel like massspectrometry and biochemical assay will help to expand the use of KTGS, and further technological development could include the use of ${ }^{19} \mathrm{~F}$-ligand-based NMR, already used for DCC, as a detection method. ${ }^{63}$ Finally, as the success of KTGS depends also on the diversity sets of reagents used, there is a high opportunity for shared reagents libraries to expand KTGS use and the size of experiments. Precompetitive collaboration in this area is thus highly desirable.

In all, this review and the in-depth analysis of all published examples demonstrated the robustness and versatility of KTGS and its utility as a method combining in a single step diversity generation and selection method in the three stages of drug discovery: hit discovery, hit-to-lead and lead optimization.

\section{AUTHOR INFORMATION}

\section{Corresponding Author}

*Phone:. +33.320.964.948 Email: rebecca.deprez@univ-lille.fr ;ORCID \# 0000-0002-3318-5297 


\section{Notes}

The authors declare no competing financial interest. We thank Julie Kerr-Conte for proof-reading.

\section{ABBREVIATIONS USED}

Ac, Acinetobacter calcoaceticus; ; AChBP, Acetylcholine binding protein; AchE, Acetylcholinesterase; ADME, Absorption, Distribution, Metabolism, and Excretion; ATP, Adenosine Tri-Phosphate; AUC , Area Under the Curve; BACE-1, $\beta$-site amyloid precursor protein cleaving enzyme 1; BAX, Bcl-2-associated X protein; Bcl-XL, B-cell lymphoma extra-large protein; BPL, Biotin Protein Ligase; bRo5, beyond Rule of 5; CAll, Carbonic Anhydrase II; CAIl, Carbonic Anhydrase II ; CETSA, Cellular Thermal Shift Assay; CLEC, C-type lectin domain; CNS, Central Nervous System; COX-2, cyclooxygenase -2; c-SRC , cellular sarcoma proto-oncogene tyrosine kinase; c-SRC, Proto-oncogene tyrosine-protein kinase Src; DAO, D-Amino-acid Oxydase; DCC, Dynamic combinatorial chemistry; DDF, dideazafolate; DIOS, Desorption/ionization on silicon; DOPA, 3,4-dihydroxyphenylalanine; EthR, EthA transcriptional repressor; EV-A71 3Cpro, Enterovirus A71 3C protease; FAD, Flavin adenine dinucleotide; FBDD, Fragment-based drug discovery; FCsp3, Fraction of sp3 hybridized carbon atoms; FDA, Food \& Drug Administration; FLT3-ITD, Fms-like tyrosine kinase-internal tandem duplication mutations; FLuc, firefly luciferase; GARTfase, Glycinamide ribonucleotide transformylase; HBA, number of Hydrogen-Bond Acceptors; HBD, number of Hydrogen-Bond Donors; HCA, Human Colon Adenocarcinoma; HPLC, High Performance Liquid Chromatography; HRMS, High Resolution Mass Spectrometry; HRTOF, High Resolution Time Of Flight; HRV, Human Rhinovirus; IDE, Insulin-Degrading Enzyme; iPrOH, Isopropanol; ITDRF, Isothermal dose-response fingerprint; ko, knock-out; Kp, Klebsiella pneumoniae; KTGS, Kinetic Target-Guided Synthesis; LCMS, Tandem Liquid chromatography-mass spectrometry; LE, Ligand Efficiency; LLE, Lipophilic Ligand Efficiency; Lm, Listeria monocytogenes ; MAO-B , Monoamine oxidase-B; MBP, Maltose binding protein; Mcl 1, Myeloid leukemia cell differentiation protein; MCR: multicomponent reaction; MIC, Minimum Inhibitory Concentration ; MIF, Macrophage migration inhibitory factor; MRM, Multiple Reaction Monitoring; MS, Mass Spectrometry; Mtb, Mycobacterium tuberculosis; MW, Molecular Weight; nAChRs, Nicotinic acetylcholine receptors; NAD, Nicotinamide Adenine Dinucleotide; NADK1, NAD kinase -1; NBD, 4-chloro-7-nitro-1,2,3benzoxadiazole; nRot, Number of rotatable bonds; OC50, Occupancy concentration 50; O-GlcNAc, N- 
acetylglucosamine; OGT, O-linked $\beta$-N-acetylglucosamine transferase; PET, Positron emission tomography; po, per os; PPI, Protein-Protein Interaction; PSA, Polar Surface Area; PTCC, Proteintemplated click chemistry; PTS, Protein-templated synthesis ; RBC, Red Blood Cells; Ro3, Rule of 3; Ro5, Rule of 5; S. aureus, Staphylococcus aureus; SAXS, Small-Angle X-ray Scattering ; sc, subcutaneous; STAT5, Signal Transducer and Activator of Transcription 5; TGS, Target-guided synthesis; TISCC, Tethering-in situ click chemistry ; UDP-GlcNAc, Uridine diphosphate Nacetylglucosamine; wt, wild type.

\section{ASSOCIATED CONTENT:}

The Supporting Information is available free of charge on the ACS Publications website at DOI: XXX Supplementary Tables with Pairs of warhead-bearing reagents and corresponding KTGS ligand structures; Full practical details of published KTGS examples. Supplementary Figures of 3D-Plots of parameters for FDA and KTGS ligands and Distribution and Box plots of MW, CLogP, HBD, HBA, rotatable bonds and PSA for KTGS ligands vs FDA approved compounds; Supplementary methods.

\section{Biographies}

Damien Bosc obtained an Engineer Degree from INP-Toulouse (ENSIACET), a M. Sc. in Organic Chemistry (Toulouse, FR) in 2008. He received his Ph.D. from the University of Paris-Sud, FR in 2011 within the group of Dr Dubois at ICSN (Gif-sur-Yvette, FR). He held postdoc positions in the groups of Pr Deprez at the School of Pharmacy in Lille, FR, Prof. R. N. Young at the Simon Fraser University, Vancouver, CA, and of Pr Hibert and Dr. Bonnet at the School of Pharmacy in Strasbourg, FR. He has worked on metalloenzymes, proteases and chemokine receptors. Since 2016, he is assistant professor in Medicinal Chemistry at the School of Pharmacy in Lille. His research interests include medicinal chemistry and in-situ click chemistry. ORCID \#0000-0002-6076-2934

Virgyl Camberlein graduated from the School of Pharmacy in Lille, FR in 2018 and obtained his M. Sc. in Medicinal Chemistry the same year, on the design and optimization of metalloprotease inhibitors. He is currently doing his $\mathrm{PhD}$ on protein-templated reactions. 
Omar Castillo-Aguilera graduated with a degree in Pharmacy from BUAP, Mexico, in 2011. He obtained his MSc in Drug Design from Univ. Lille (FR) in 2014 with a thesis focusing on the development of CAIX inhibitors. In 2017, he obtained his PhD under the supervision of P. Depreux (ICPAL, Univ. Lille, FR) and P.B. Arimondo (ETaC, CNRS-Pierre Fabre, FR) on the development of epigenetic inhibitors. He then carried out a postdoc in the group of S. Comesse (Le Havre, FR), on the development of domino reactions. In 2018, he joined the team of R. Deprez-Poulain as a postdoc to work on the development of metalloprotease inhibitors.

Ronan Gealageas got his MSc in Drug Design and Combinatorial Chemistry in 2006 (Université d'Orléans, FR). After working for 2 years in Dr. Bourguignon's (IIlkirch, FR) on RPFF inhibitors, he did his $\mathrm{PhD}$ on bioluminescent probes for in vivo imaging and inhibitors of Dyrk1a, in the team of Dr. R. Dodd at the ICSN (Gif-sur-Yvette, FR). After his PhD (2011), he did a two-year postdoc in Pr. Grierson's lab (Univ. British Columbia, Vancouver, CA) on gut-directed MAO-B inhibitors. He has been working on modulators of metalloproteases, click-chemistry and KTGS in the team of Pr. Deprez-Poulain since 2015.

Benoit Deprez, MBA (EDHEC), pharmacist, PhD was previously director of chemistry at CEREP and head of the drug discovery team at Devgen (Ghent, BE). In 2003, he was appointed professor at the School of Pharmacy of Lille, FR. He created and heads the drug discovery lab INSERM U1177 (Univ Lille and Institut Pasteur de Lille). He co-founded the biotech APTEEUS. He has managed several industrial collaborations and 2 molecules resulting from a partnership with Bioversys and GSK have entered into development. He is corresponding member of the National Academy of Pharmacy and scientific director of the Institut Pasteur de Lille. He is the recipient of the 2017 Pierre Fabre Award for therapeutic innovation. He is the author of more than 100 papers and 24 patents. ORCID \#0000-0002$2777-4538$

Rebecca Deprez-Poulain holds a BS in Chemistry (Juniata College, PA), and an engineer degree, (HEI, Lille, FR). She obtained her PhD under the supervision of Pr Tartar on combinatorial chemistry at CEREP (1999) and her PharmD at the School of Pharmacy in Lille where she is now Full Professor of Medicinal Chemistry. She created the mobile application "MedChemStructures Genius". She has been 
awarded the joined French Medicinal Chemistry Society-Servier Prize (2009) and is a member of the Institut Universitaire de France (2015). Her team is part of the drug discovery lab INSERM U1177 (Univ Lille and Institut Pasteur de Lille). She has published more than 60 papers. Her recent research interests include the use of kinetic target guided synthesis to identify drug-like inhibitors of metalloenzymes. ORCID \#0000-0002-3318-5297

\section{REFERENCES}

(1) Jaegle, M.; Wong, E. L.; Tauber, C.; Nawrotzky, E.; Arkona, C.; Rademann, J. ProteinTemplated Fragment Ligations_-from Molecular Recognition to Drug Discovery. Angew. Chem. Int. Ed. 2017, 56, 7358-7378.

(2) Bosc, D.; Jakhlal, J.; Deprez, B.; Deprez-Poulain, R. Kinetic Target-Guided Synthesis in Drug Discovery and Chemical Biology: A Comprehensive Facts and Figures Survey. Future Med. Chem. 2016, 8, 381-404.

(3) Wu, G.; Zhao, T.; Kang, D.; Zhang, J.; Song, Y.; Namasivayam, V.; Kongsted, J.; Pannecouque, C.; De Clercq, E.; Poongavanam, V.; Liu, X.; Zhan, P. Overview of Recent Strategic Advances in Medicinal Chemistry. J. Med. Chem. 2019.in press DOI: 10.1021/acs.jmedchem.9b00359

(4) Cheeseman, J. D.; Corbett, A. D.; Gleason, J. L.; Kazlauskas, R. J. Receptor-Assisted Combinatorial Chemistry: Thermodynamics and Kinetics in Drug Discovery. Chem. Eur. J. 2005, 11, 1708-1716.

(5) Hartman, A. M.; Gierse, R. M.; Hirsch, A. K. H. Protein-Templated Dynamic Combinatorial Chemistry: Brief Overview and Experimental Protocol. Eur. J. Org. Chem. 2019, 2019, 3581-3590.

(6) Frei, P.; Hevey, R.; Ernst, B. Dynamic Combinatorial Chemistry: A New Methodology Comes of Age. Chemistry 2019, 25, 60-73.

(7) Hu, X.; Manetsch, R. Kinetic Target-Guided Synthesis. Chem. Soc. Rev. 2010, 39, 1316-1324.

(8) Oueis, E.; Sabot, C.; Renard, P.-Y. New Insights into the Kinetic Target-Guided Synthesis of Protein Ligands. Chem. Commun. 2015, 51, 12158-12169.

(9) Lewis, W. G.; Green, L. G.; Grynszpan, F.; Radić, Z.; Carlier, P. R.; Taylor, P.; Finn, M. G.; Sharpless, K. B. Click Chemistry in Situ: Acetylcholinesterase as a Reaction Vessel for the Selective 
Assembly of a Femtomolar Inhibitor from an Array of Building Blocks. Angew. Chem. Int. Ed. 2002, 41, 1053-1057.

(10) Congreve, M.; Carr, R.; Murray, C.; Jhoti, H. A 'Rule of Three' for Fragment-Based Lead Discovery? Drug Discov. Today 2003, 8, 876-877.

(11) Köster, H.; Craan, T.; Brass, S.; Herhaus, C.; Zentgraf, M.; Neumann, L.; Heine, A.; Klebe, G. A Small Nonrule of 3 Compatible Fragment Library Provides High Hit Rate of Endothiapepsin Crystal Structures with Various Fragment Chemotypes. J. Med. Chem. 2011, 54, 7784-7796.

(12) Inglese, J.; Benkovic, S. J. Multisubstrate Adduct Inhibitors of Glycinamide Ribonucleotide Transformylase: Synthetic and Enzyme-Assembled. Tetrahedron 1991, 47, 2351-2364.

(13) Potashman, M. H.; Duggan, M. E. Covalent Modifiers: An Orthogonal Approach to Drug Design. J. Med. Chem. 2009, 52, 1231-1246.

(14) Auld, D. S.; Lovell, S.; Thorne, N.; Lea, W. A.; Maloney, D. J.; Shen, M.; Rai, G.; Battaile, K. P.; Thomas, C. J.; Simeonov, A.; Hanzlik, R. P.; Inglese, J. Molecular Basis for the High-Affinity Binding and Stabilization of Firefly Luciferase by PTC124. PNAS 2010, 107, 4878-4883.

(15) Nguyen, R.; Huc, I. Using an Enzyme's Active Site to Template Inhibitors. Angew. Chem. Int. Ed. 2001, 40, 1774-1776.

(16) Rostovtsev, V. V.; Green, L. G.; Fokin, V. V.; Sharpless, K. B. A Stepwise Huisgen Cycloaddition Process: Copper(I)-Catalyzed Regioselective "Ligation" of Azides and Terminal Alkynes. Angew. Chem. Int. Ed. 2002, 41, 2596-2599.

(17) Tornøe, C. W.; Christensen, C.; Meldal, M. Peptidotriazoles on Solid Phase: [1,2,3]-Triazoles by Regiospecific Copper(I)-Catalyzed 1,3-Dipolar Cycloadditions of Terminal Alkynes to Azides. J. Org. Chem. 2002, 67, 3057-3064.

(18) Willand, N.; Desroses, M.; Toto, P.; Dirié, B.; Lens, Z.; Villeret, V.; Rucktooa, P.; Locht, C.; Baulard, A.; Deprez, B. Exploring Drug Target Flexibility Using in Situ Click Chemistry: Application to a Mycobacterial Transcriptional Regulator. ACS Chem. Biol. 2010, 5, 1007-1013.

(19) Wong, E. L.; Nawrotzky, E.; Arkona, C.; Kim, B. G.; Beligny, S.; Wang, X.; Wagner, S.; Lisurek, M.; Carstanjen, D.; Rademann, J. The Transcription Factor Stat5 Catalyzes Mannich Ligation Reactions Yielding Inhibitors of Leukemic Cell Proliferation. Nat. Commun. 2019, 10, 66.

(20) Grimster, N. P.; Stump, B.; Fotsing, J. R.; Weide, T.; Talley, T. T.; Yamauchi, J. G.; Nemecz, Ã. k.; Kim, C.; Ho, K.-Y.; Sharpless, K. B.; Taylor, P.; Fokin, V. V. Generation of Candidate Ligands for 
Nicotinic Acetylcholine Receptors Via in Situ Click Chemistry with a Soluble Acetylcholine Binding Protein Template. J. Am. Chem. Soc. 2012, 134, 6732-6740.

(21) Kulkarni, S. S.; Hu, X.; Doi, K.; Wang, H.-G.; Manetsch, R. Screening of Protein-Protein Interaction Modulators Via Sulfo-Click Kinetic Target-Guided Synthesis. ACS Chem. Biol. 2011, 6, 724732.

(22) Unver, M. Y.; Gierse, R. M.; Ritchie, H.; Hirsch, A. K. H. Druggability Assessment of Targets Used in Kinetic Target-Guided Synthesis. J. Med. Chem. 2018, 61, 9395-9409.

(23) Oueis, E.; Nachon, F.; Sabot, C.; Renard, P.-Y. First Enzymatic Hydrolysis/Thio-Michael Addition Cascade Route to Synthesis of AChE Inhibitors. Chem. Commun. 2014, 50, 2043-2015.

(24) Jaegle, M.; Steinmetzer, T.; Rademann, J. Protein-Templated Formation of an Inhibitor of the Blood Coagulation Factor Xa through a Background-Free Amidation Reaction. Angew. Chem. Int. Ed. 2017, 56, 3718-3722.

(25) Wang, Y.; Zhu, J.; Zhang, L. Discovery of Cell-Permeable O-Glcnac Transferase Inhibitors Via Tethering in Situ Click Chemistry. J. Med. Chem. 2017, 60, 263-272.

(26) Stürzebecher, A.; Dönnecke, D.; Schweinitz, A.; Schuster, O.; Steinmetzer, P.; Stürzebecher, U.; Kotthaus, J.; Clement, B.; Stürzebecher, J.; Steinmetzer, T. Highly Potent and Selective Substrate Analogue Factor Xa Inhibitors Containing D-Homophenylalanine Analogues as P3 Residue: Part 2. ChemMedChem 2007, 2, 1043-1053.

(27) Boström, J.; Brown, D. G.; Young, R. J.; Keserü, G. M. Expanding the Medicinal Chemistry Synthetic Toolbox. Nat. Rev. Drug Discovery 2018, 17, 709-727.

(28) Gelin, M.; Poncet-Montange, G.; Assairi, L.; Morellato, L.; Huteau, V.; Dugue, L.; Dussurget, O.; Pochet, S.; Labesse, G. Screening and in Situ Synthesis Using Crystals of a NAD Kinase Lead to a Potent Antistaphylococcal Compound. Structure 2012, 20, 1107-1117.

(29) a) Weber, L. In Vitro Combinatorial Chemistry to Create Drug Candidates. Drug Discovery Today: Technologies 2004, 1, 261-267. b) Weber, L. and D'Arcy, A. Methods in combinatorial chemistry. Proceedings of the 17th Regio-Symposium, Sornetan, Switzerland, September 24-26 1997.

(30) Bhardwaj, A.; Kaur, J.; Wuest, M.; Wuest, F. In Situ Click Chemistry Generation of Cyclooxygenase-2 Inhibitors. Nat. Commun. 2017, 8, 1.

(31) Deprez-Poulain, R.; Hennuyer, N.; Bosc, D.; Liang, W. G.; Enée, E.; Marechal, X.; Charton, J.; Totobenazara, J.; Berte, G.; Jahklal, J.; Verdelet, T.; Dumont, J.; Dassonneville, S.; Woitrain, E.; Gauriot, 
M.; Paquet, C.; Duplan, I.; Hermant, P.; Cantrelle, F.-X.; Sevin, E.; Culot, M.; Landry, V.; Herledan, A.; Piveteau, C.; Lippens, G.; Leroux, F.; Tang, W.-J.; van Endert, P.; Staels, B.; Deprez, B. Catalytic Site Inhibition of Insulin-Degrading Enzyme by a Small Molecule Induces Glucose Intolerance in Mice. Nat. Commun. 2015, 6, 8250.

(32) Krasiński, A.; Radić, Z.; Manetsch, R.; Raushel, J.; Taylor, P.; Sharpless, K. B.; Kolb, H. C. In Situ Selection of Lead Compounds by Click Chemistry: Target-Guided Optimization of Acetylcholinesterase Inhibitors. J. Am. Chem. Soc. 2005, 127, 6686-6692.

(33) Oueis, E.; Santoni, G.; Ronco, C.; Syzgantseva, O.; Tognetti, V.; Joubert, L.; Romieu, A.; Weik, M.; Jean, L.; Sabot, C.; Nachon, F.; Renard, P.-Y. Reaction Site-Driven Regioselective Synthesis of AChE Inhibitors. Org. Biomol. Chem. 2014, 12, 156.

(34) Paparella, A. S.; Feng, J.; Blanco-Rodriguez, B.; Feng, Z.; Phetsang, W.; Blaskovich, M. A. T.; Cooper, M. A.; Booker, G. W.; Polyak, S. W.; Abell, A. D. A Template Guided Approach to Generating Cell Permeable Inhibitors of Staphylococcus Aureus Biotin Protein Ligase. Tetrahedron 2018, 74, 11751183.

(35) Tieu, W.; Soares da Costa, T. P.; Yap, M. Y.; Keeling, K. L.; Wilce, M. C. J.; Wallace, J. C.; Booker, G. W.; Polyak, S. W.; Abell, A. D. Optimising in Situ Click Chemistry: The Screening and Identification of Biotin Protein Ligase Inhibitors. Chemical Science 2013, 4, 3533-3537.

(36) Rzuczek, S. G.; Park, H.; Disney, M. D. A Toxic RNA Catalyzes the in Cellulo Synthesis of Its Own Inhibitor. Angew. Chem. Int. Ed. 2014, 53, 10956-10959.

(37) Jin, X.; Daher, S. S.; Lee, M.; Buttaro, B.; Andrade, R. B. Ribosome-Templated Azide-Alkyne Cycloadditions Using Resistant Bacteria as Reaction Vessels: In Cellulo Click Chemistry. ACS Med. Chem. Lett. 2018, 9, 907-911.

(38) Fu, S.; Nie, Q.; Ma, Y.; Song, P.; Ren, X.; Luo, C.; Shang, L.; Yin, Z. Target-Guided Screening of Fragments (TGSOF) in the Discovery of Inhibitors against Ev-A71 3c Protease. Chem. Commun. 2018, 54, 2890-2893.

(39) Mondal, M.; Unver, M. Y.; Pal, A.; Bakker, M.; Berrier, S. P.; Hirsch, A. K. H. Fragment-Based Drug Design Facilitated by Protein-Templated Click Chemistry: Fragment Linking and Optimization of Inhibitors of the Aspartic Protease Endothiapepsin. Chem. Eur. J. 2016, 22, 14826-14830. 
(40) Shelke, S. V.; Cutting, B.; Jiang, X.; Koliwer-Brandl, H.; Strasser, D. S.; Schwardt, O.; Kelm, S.;

Ernst, B. A Fragment-Based in Situ Combinatorial Approach to Identify High-Affinity Ligands for Unknown Binding Sites. Angew. Chem. Int. Ed. 2010, 49, 5721-5725.

(41) Hirose, T.; Sunazuka, T.; Sugawara, A.; Endo, A.; Iguchi, K.; Yamamoto, T.; Ui, H.; Shiomi, K.; Watanabe, T.; Sharpless, K. B.; Omura, S. Chitinase Inhibitors: Extraction of the Active Framework from Natural Argifin and Use of in Situ Click Chemistry. J Antibiot 2009, 62, 277-282.

(42) Giordanetto, F.; Jin, C.; Willmore, L.; Feher, M.; Shaw, D. E. Fragment Hits: What Do They Look Like and How Do They Bind? J. Med. Chem. 2019, 62, 3381-3394.

(43) Kwarcinski, F. E.; Steffey, M. E.; Fox, C. C.; Soellner, M. B. Discovery of Bivalent Kinase Inhibitors Via Enzyme-Templated Fragment Elaboration. ACS Med. Chem. Lett. 2015, 6, 898-901.

(44) Toguchi, S.; Hirose, T.; Yorita, K.; Fukui, K.; Sharpless, K. B.; Ōmura, S.; Sunazuka, T. In Situ Click Chemistry for the Identification of a Potent D-Amino Acid Oxidase Inhibitor. Chem. Pharm. Bull. (Tokyo). 2016, 64, 695-703.

(45) Wang, Y.; Lin, W.-Y.; Liu, K.; Lin, R. J.; Selke, M.; Kolb, H. C.; Zhang, N.; Zhao, X.-Z.; Phelps, M. E.; Shen, C. K. F.; Faull, K. F.; Tseng, H.-R. An Integrated Microfluidic Device for Large-Scale in Situ Click Chemistry Screening. Lab Chip 2009, 9, 2281-2285.

(46) Antti, H.; Sellstedt, M. Cell-Based Kinetic Target-Guided Synthesis of an Enzyme Inhibitor. ACS Med. Chem. Lett. 2018, 9, 351-353.

(47) Parvatkar, P.; Kato, N.; Uesugi, M.; Sato, S.-i.; Ohkanda, J. Intracellular Generation of a Diterpene-Peptide Conjugate That Inhibits 14-3-3-Mediated Interactions. J. Am. Chem. Soc. 2015, 137, 15624-15627.

(48) Statsuk, A. V.; Maly, D. J.; Seeliger, M. A.; Fabian, M. A.; Biggs, W. H.; Lockhart, D. J.; Zarrinkar, P. P.; Kuriyan, J.; Shokat, K. M. Tuning a Three-Component Reaction for Trapping Kinase Substrate Complexes. J. Am. Chem. Soc. 2008, 130, 17568-17574.

(49) Wang, Y.; Cao, L.; Zhai, Y.; Yin, Z.; Sun, Y.; Shang, L. Structure of the Enterovirus 71 3c Protease in Complex with Nk-1.8k and Indications for the Development of Antienterovirus Protease Inhibitor. Antimicrob. Agents Chemother. 2017, 61, e00298-17.

(50) Glassford, I.; Teijaro, C. N.; Daher, S. S.; Weil, A.; Small, M. C.; Redhu, S. K.; Colussi, D. J.; Jacobson, M. A.; Childers, W. E.; Buttaro, B.; Nicholson, A. W.; MacKerell, A. D.; Cooperman, B. S.; 
Andrade, R. B. Ribosome-Templated Azide-Alkyne Cycloadditions: Synthesis of Potent Macrolide Antibiotics by in Situ Click Chemistry. J. Am. Chem. Soc. 2016, 138, 3136-3144.

(51) Mocharla, V. P.; Walsh, J. C.; Padgett, H. C.; Su, H.; Fueger, B.; Weber, W. A.; Czernin, J.; Kolb, H. C. From in Situ to in Vivo: An in Situ Click-Chemistry-Derived Carbonic Anhydrase li Imaging Agent for Positron Emission Tomography. ChemMedChem 2013, 8, 43-48.

(52) Hermant, P.; Bosc, D.; Piveteau, C.; Gealageas, R.; Lam, B. V.; Ronco, C.; Roignant, M.; Tolajanahary, H.; Jean, L.; Renard, P.-Y.; Lemdani, M.; Bourotte, M.; Herledan, A.; Bedart, C.; Biela, A.; Leroux, F.; Deprez, B.; Deprez-Poulain, R. Controlling Plasma Stability of Hydroxamic Acids: A Medchem Toolbox. J. Med. Chem. 2017, 60, 9067-9089.

(53) Kolb, H.C.; Mocharla, V.P.; Walsh, J.C. A method for the preparation of imaging probes using click chemistry, WO2006116736A2, April 27, 2006.

(54) Marzinzik, A.; Voegtle, M.; Rondeau, J.-M.; Billich, A.; Pelle, X.; Manetsch, R.; Monastyrskyi, A. Kinetic Target Guided Synthesis for the Identification of Macrophage Migration Inhibitory Factor Inhibitors (Mif). https://oak.novartis.com/21010/ (accessed November 13, 2019).

(55) Manetsch, R.; Nacheva, K.; Flanigan, D.; Namelikonda, N.; Iyamu, I.; Kulkarni, S.; Barber, M.; Tipton, J.; Wang, H.-G.; Doi, K. Target Binding Molecules Identified by Kinetic Target-Guided Synthesis. US. Pat. Appl. 2016116482 (A1), April 28, 2016.

(56) Palomo, V.; Garcia-Rubia, A.; Nozal-Garcia, V.; Roca, C.; Pérez, C.; Martinez, A. in situ-click chemistry: an ideal target based approach for the generation of multitarget drugs. Proceedings of the EFMC-ISMC, Llubjana, SL, September 2-6 2018.

(57) Masini, T.; Kroezen, B. S.; Hirsch, A. K. H. Druggability of the Enzymes of the Non-MevalonatePathway. Drug Discov. Today 2013, 18, 1256-1262.

(58) Zhang, Z.; Liang, W. G.; Bailey, L. J.; Tan, Y. Z.; Wei, H.; Wang, A.; Farcasanu, M.; Woods, V. A.; McCord, L. A.; Lee, D.; Shang, W.; Deprez-Poulain, R.; Deprez, B.; Liu, D. R.; Koide, A.; Koide, S.; Kossiakoff, A. A.; Li, S.; Carragher, B.; Potter, C. S.; Tang, W.-J. Ensemble Cryoem Elucidates the Mechanism of Insulin Capture and Degradation by Human Insulin Degrading Enzyme. eLife 2018, 7, e33572.

(59) Bourne, Y.; Sharpless, K. B.; Taylor, P.; Marchot, P. Steric and Dynamic Parameters Influencing in Situ Cycloadditions to Form Triazole Inhibitors with Crystalline Acetylcholinesterase. J. Am. Chem. Soc. 2016, 138, 1611-1621. 
(60) Doak, Bradley C.; Over, B.; Giordanetto, F.; Kihlberg, J. Oral Druggable Space Beyond the Rule of 5: Insights from Drugs and Clinical Candidates. Chemistry \& Biology 2014, 21, 1115-1142.

(61) Gehringer, M.; Laufer, S. A. Emerging and Re-Emerging Warheads for Targeted Covalent Inhibitors: Applications in Medicinal Chemistry and Chemical Biology. J. Med. Chem. 2019, 62, 56735724.

(62) Gladysz, R.; Vrijdag, J.; Van Rompaey, D.; Lambeir, A.-M.; Augustyns, K.; De Winter, H.; Van der Veken, P. Efforts Towards an on-Target Version of the Groebke-Blackburn-Bienaymé (GBB) Reaction for Discovery of Druglike Urokinase (UPA) Inhibitors. Chem. Eur. J. 2019, 25, 12380-12393.

(63) Ekström, A. G.; Wang, J. T.; Bella, J.; Campopiano, D. J. Non-Invasive ${ }^{19} \mathrm{~F}$ NMR Analysis of a Protein-Templated N-Acylhydrazone Dynamic Combinatorial Library. Org. Biomol. Chem. 2018, 16, 8144-8149. 
Table of content Graphic

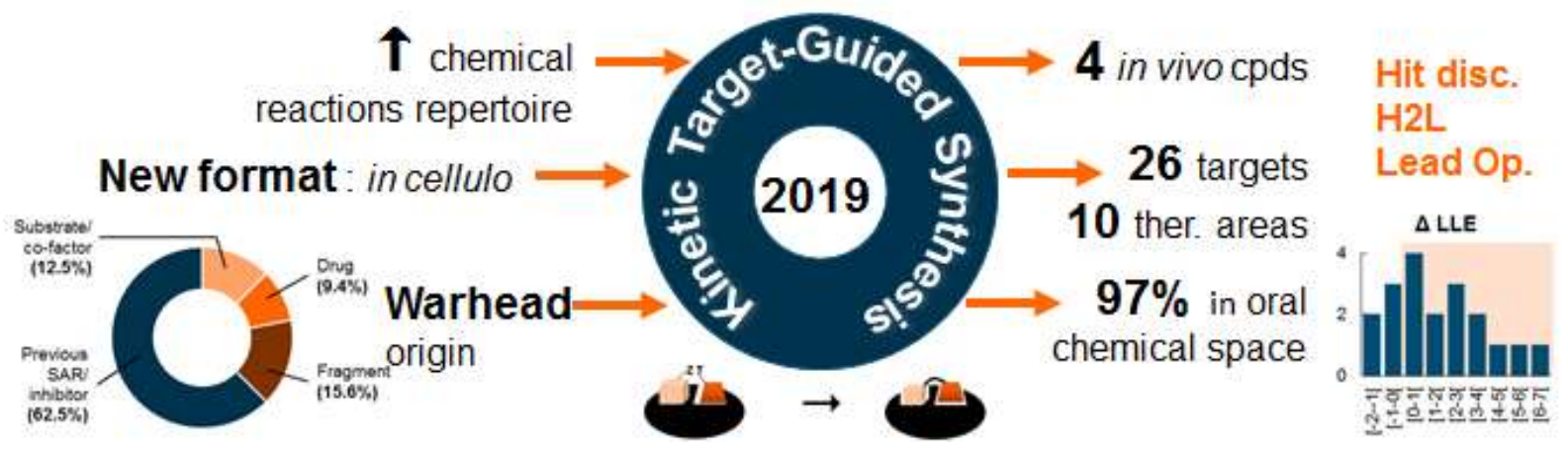

